\title{
INTEGRAL REPRESENTATIONS OF POSITIVE DEFINITE MATRIX-VALUED DISTRIBUTIONS ON CYLINDERS
}

\author{
JÜRGEN FRIEDRICH
}

\begin{abstract}
The notion of a $G$-continuous matrix-valued positive definite distribution on $S_{N}(2 a) \times \mathbf{R}^{M} \times G$ is introduced, where $G$ is an abelian separable locally compact group and where $S_{N}(2 a)$ is an open ball around zero in $\mathbf{R}^{N}$ with radius $2 a>0$. This notion generalizes that one of strongly continuous positive definite operator-valued functions. For these objects, a Bochner-type theorem gives a suitable integral representation if $N=1$ or if the matrix-valued distribution is invariant w.r.t. rotations in $\mathbf{R}^{N}$. As a consequence, appropriate extensions to the whole group are obtained. In particular, we show that a positive definite function on a certain cylinder in a separable real Hilbert space $H$ may be extended to a characteristic function of a finite positive measure on $H$, if it is invariant w.r.t. rotations and continuous w.r.t. a suitable topology.
\end{abstract}

\section{INTRODUCTION}

In 1939 M. G. Krein [12] considered the following problem: Are there positive definite extensions of a continuous positive definite function on an interval $(-2 a, 2 a), 0<a \leq \infty$, to the whole real axis? He answered this question in the affirmative. Rudin showed in [20] that there is no positive definite extension of a continuous positive definite function given on a square to the whole plane in general. Various papers are related to the extendability of continuous positive definite (also operator-valued) functions from subsets of the form $(-2 a, 2 a) \times G$ to the whole group $\mathrm{R} \times G$, where $G$ is a suitable abelian topological group (cf. e.g. $[15,4,9,14,7])$.

Despite the fact that there are no extensions of continuous positive definite functions from squares to the plane in general, Rudin [21] proved that an extension is always possible, if the function in question is given on an open ball around zero and if it is invariant w.r.t. rotations. Nussbaum extended this result to distributions $[17,18]$. More precisely, he obtained a representation of the given distribution as an integral over certain elementary positive definite distributions. The latter ones may be extended to the whole space in an obvious way. Thus the appropriate integral representation describes an extension of the original distribution. Conversely, the Bochner-Schwartz Theorem yields an appropriate integral representation for each positive definite extension of the

Received by the editors December 17, 1987.

1980 Mathematics Subject Classification (1985 Revision). Primary 43A35, 42A82; Secondary 60B15, 47A20. 
original distribution i.e., integral representations and positive definite extensions are intimately related.

Generalizing the objects under consideration w.r.t. their ranges one is led to functions taking sesquilinear forms over complex (topological) vector spaces as values. Nevertheless, this situation is easily reduced to the case of functions which values are bounded operators in a Hilbert space [7, Lemma 1].

The aim of this paper is to show the extendability of such positive definite operator-valued functions $F$ defined on a stratum or on a cylindrical subset of an abelian topological group to the whole group. In the latter case we assume that $F$ is invariant w.r.t. all rotations leaving the cylinder invariant.

To prove this result we follow essentially the scheme in [17]. Especially, we want to use the nuclear spectral theorem to obtain a suitable integral representation. To bring nuclearity into play it is more useful to consider the matrix representation of $F$ w.r.t. a fixed basis in the Hilbert space instead of the function $F$ itself. For the proof it is almost equal, whether we consider matrices of continuous functions or even of distributions. Therefore we introduce $G$-continuous positive definite matrix-valued distributions $\mathscr{F}$ on $S_{N}(2 a) \times \mathbb{R}^{M} \times G$, where $S_{N}(2 a)$ is the open ball around zero in $\mathbb{R}^{N}$ with radius $2 a>0$ and where $G$ is a separable locally compact abelian group (Definition 3 ). The entries of the infinite matrix $\mathscr{F}$ are distributions on $S_{N}(2 a) \times \mathbb{R}^{M}$, depending continuously on the additional parameter $g \in G$. If $N=1$ or if all entries are invariant w.r.t. rotations in $\mathbb{R}^{N}$, we obtain a Bochner-like representation of $\mathscr{F}$ as the Fourier transform of some nonnegative matrix-valued measure. This representation implies the extendability of the appropriate matrix-valued distributions to the whole group (Theorems 1 and 2). As a consequence, we can prove the existence of positive definite extensions of operator-valued positive definite functions on strata or cylinders (described above) to the whole group even if the latter is not locally compact. In particular, we show that a positive definite function on the cylinder $S_{N}(2 a) \times H_{2}$ in the real separable Hilbert space $H=\mathbb{R}^{N} \times H_{2}$ may be extended to a characteristic function of a finite positive Borel measure on $H$, if it is invariant w.r.t. rotations in $H_{1}=\mathbb{R}^{N}$ and continuous w.r.t. to a suitable topology. A similar result for positive definite functions on a stratum was already obtained in [3].

The paper is organized as follows. In $\S \S 3$ and 4 we consider simple facts about positive definite operator-valued functions and matrix-valued distributions. In $\S 5$ we introduce certain auxiliary nuclear spaces and transfer positive definiteness to positivity of certain linear functionals. In $\S 6$ we note a version of the nuclear spectral theorem, which is used later on. Then we discuss extensions of symmetric operators commuting with unitary representations of an abelian group and appropriate direct integral Hilbert spaces. This provides the operator theoretic background for the extensions of positive definite functions. $\S \S 9$ and 10 we deduce our main results and appropriate conclusions. 


\section{Notations}

The letters $\mathbb{N}\left(\mathbf{R}, \mathbf{R}_{+}, \mathbb{C}\right)$ denote the sets of positive integers (real numbers, nonnegative real numbers, complex numbers). For a locally convex space $E$, $E^{\prime}$ denotes the vector space of continuous linear functionals on $E$. The value of some $e^{\prime} \in E^{\prime}$ at $e \in E$ is written $e^{\prime}(e)$. The space of all matrices $\left(e_{k l}\right)_{k, l=1}^{n}$, $n \in \mathbb{N} \cup\{\infty\}, e_{k l} \in E$, is denoted by $M(n ; E)$. The spaces $M(n ; E)$, $n \in \mathbb{N}$, are considered as subspaces of $M(\infty ; E)$ in the usual way. The subspace $\bigcup_{n \in \mathbb{N}} M(n ; E)$ of $M(\infty ; E)$ is denoted by $M(\omega ; E)$. If $E=\mathbb{C}$, we write simply $M(n)$ and $M(\omega)$ instead of $M(n ; \mathbb{C})$ and $M(\omega ; \mathbb{C}), n \in$ $\mathbb{N} \cup\{\infty\}$. If $x=\left(x_{1}, \ldots, x_{N}\right)$ and $\xi=\left(\xi_{1}, \ldots, \xi_{N}\right)$ are points in $\mathbf{R}^{N}$, $N \in \mathrm{N}$, we abbreviate $x \cdot \xi=x_{1} \xi_{1}+\cdots+x_{N} \xi_{N},|x|=(x \cdot x)^{1 / 2}$, and $S_{N}(a)=$ $\left\{x \in \mathbf{R}^{N} ;|x|<a\right\}, 0<a \leq \infty$. We define $\mathbf{R}^{0}=\{0\}$. The letter $G$ is always used for an abelian group. Group operations are written additively and 0 denotes the natures element. If $G$ is locally compact, $G^{*}$ denotes the dual group of $G$. The value of $\gamma \in G^{*}$ at $g \in G$ is $[\gamma, g]$. Note that $\left(\mathbb{R}^{N}\right)^{*}$ is isomorphic to $\mathbb{R}^{N}$. For $x \in \mathbb{R}^{N}$ and $\xi \in\left(\mathbf{R}^{N}\right)^{*}=\mathbf{R}^{N}$ we assume that $[\xi, x]=\exp (-i \xi \cdot x)$. Let $O(N)$ denote the orthogonal group of $\mathbf{R}^{N}$ and $d \rho$ the Haar measure on $O(N)$, normalized such that $\int_{O(N)} d \rho=1$. The image of $\rho \in O(N)$ applied to $x \in \mathbb{R}^{N}$ is $\rho x$. If $F$ is a mapping defined on $S_{N}(a) \times \mathbb{R}^{M} \times G$, we define $\rho F, \rho \in O(N)$, by $(\rho F)(x, y, g)=F\left(\rho^{-1} x, y, g\right)$. $F$ is called $N$-radial, if $\rho F=F$ for all $\rho \in O(N)$. Let $\mathscr{D}_{a}=\mathscr{D}\left(S_{N}(a) \times \mathbb{R}^{M}\right)$ denote the usual Schwartz space of infinitely differentiable functions on $\mathbf{R}^{N+M}$ with compact support on $S_{N}(a) \times \mathbf{R}^{M}$, endowed with the usual $L F$-topology. Some $f \in \mathscr{D}_{a}^{\prime}$ is called $N$-radial, if $\rho f=f$ for all $\rho \in O(N)$, where $\rho f$ is defined by $(\rho f)(\varphi)=f\left(\rho^{-1} \varphi\right), \varphi \in \mathscr{D}_{a}$. If $f$ is a continuous complex-valued function $S_{N}(a) \times \mathbb{R}^{M} \times G$, we define a $N$-radial function $f^{\#}$ by $f^{\#}(x, y, g)=$ $\int_{O(N)} f(\rho x, y, g) d \rho$. If $H$ is a Hilbert space, we write $\langle\cdot, \cdot\rangle$ and $\|\cdot\|$ for its inner product and its norm, resp. (We will use the same symbols for different spaces, since there is no danger of confusion.) By $D(A), \bar{A}, A^{*}$, and $\operatorname{ker}(A)$ we denote the domain, closure, adjoint, and kernel of a linear operator $A$ in $H$. The space of all bounded operators in $H$ is $B(H)$.

\section{Positive Definite functions}

Throughout this paper we consider an abelian group $\mathbf{G}$, which contains a vector space $\mathbf{R}^{N+M}$ as subgroup, i.e., $\mathbf{G}=\mathbf{R}^{N+M} \times G$, where $G$ is an abelian group. We will always have to do with subsets $\mathbf{C}$ of $\mathbf{G}$ of the form $S_{N}(a) \times$ $\mathbf{R}^{M} \times G$, which will be called cylinders. If not necessary, we will not specify the parameters and only use the letter $C$. We write briefly $2 C$ instead of $S_{N}(2 a) \times \mathbf{R}^{M} \times G$. 
Definition 1. Let $H$ denote a Hilbert space. A mapping $F: 2 \mathrm{C} \rightarrow B(H)$ is called positive definite (p.d.), if $\left(F\left(\mathbf{g}_{i}-\mathbf{g}_{j}\right)\right)_{i, j=1}^{n}$ is nonnegative in $B(H) \otimes M(n)$ for all $\mathbf{g}_{1}, \ldots, \mathbf{g}_{n} \in \mathbf{C}, n \in \mathbb{N}$.

Lemma 1. Consider a linear subspace $D$ of $H$ and a subgroup $G_{0}$ of $G$. Let $\mathbf{C}_{0}=S_{N}(a) \times \mathbb{R}^{M} \times G_{0}$ and suppose that $F: 2 \mathbf{C}_{0} \rightarrow B(H)$ satisfies

(1) $\sum_{i, j=1}^{n}\left\langle F\left(\mathbf{g}_{i}-\mathbf{g}_{j}\right) X_{i}, X_{j}\right\rangle \geq 0$

$$
\text { for all } \mathbf{g}_{1}, \ldots, \mathbf{g}_{n} \in \mathbf{C}_{0}, X_{1}, \ldots, X_{n} \in D, n \in \mathbb{N} .
$$

Then

(i) $|\langle F(\mathbf{g}) X, Y\rangle|^{2} \leq\langle F(\mathbf{0}) X, X\rangle\langle F(\mathbf{0}) Y, Y\rangle$ for $\mathbf{g} \in 2 \mathbf{C}_{0}, X, Y \in \bar{D}$.

(ii) If $\bar{D}=H$, then $F(\mathbf{g})^{*}=F(-\mathbf{g})$ and $\|F(\mathbf{g})\| \leq\|F(\mathbf{0})\|, \mathbf{g} \in 2 \mathbf{C}_{0}$. Moreover, $F$ is p.d.

The proof of Lemma 1 is easy and therefore omitted.

Lemma 2. Suppose that $G$ (hence $\mathbf{G}$ ) is a topological group. If $F: 2 \mathbf{C} \rightarrow B(H)$ is p.d. and weakly continuous at $\mathbf{0}$, then it is uniformly strongly continuous.

Proof. First consider a nonnegative $(3,3)$-matrix

$$
\mathbf{A}=\left(\begin{array}{lll}
\rho & \rho & \beta \\
\bar{\alpha} & \mu & \gamma \\
\bar{\beta} & \bar{\gamma} & \mu
\end{array}\right) .
$$

The nonnegativity of $\mathrm{A}$ implies $\rho \geq 0, \mu \geq 0, \rho \cdot \mu \geq|\alpha|^{2}, \rho \cdot \mu \geq|\beta|^{2}$, $\mu \geq|\gamma|$, and $\operatorname{det} A=\rho \cdot \mu^{2}+\alpha \bar{\beta} \gamma+\bar{\alpha} \beta \bar{\gamma}-|\alpha|^{2} \mu-|\beta|^{2} \mu-|\gamma|^{2} \rho \geq 0$. Especially, $\mu=0$ implies $\alpha=\beta=\gamma=0$. Suppose that $\mu \neq 0$. Then

$$
\begin{aligned}
|\alpha-\beta|^{2} & =|\alpha|^{2}-\bar{\alpha} \beta-\alpha \bar{\beta}+|\beta|^{2} \\
& \leq\left(\rho \cdot \mu^{2}+\alpha \bar{\beta} \gamma+\bar{\alpha} \beta \bar{\gamma}-|\gamma|^{2} \rho\right) \mu^{-1}-\bar{\alpha} \beta-\alpha \bar{\beta} \\
& =\mu^{-1}\left(\rho\left(\mu^{2}-|\gamma|^{2}\right)+\alpha \bar{\beta}(\gamma-\mu)+\bar{\alpha} \beta(\bar{\gamma}-\mu)\right) \\
& \leq \mu^{-1} \rho\left(\mu^{2}-|\gamma|^{2}\right)+2 \rho|\gamma-\mu| \\
& =\rho((\mu+|\gamma|) / \mu)(\mu-|\gamma|)+2 \rho|\gamma-\mu| \\
& \leq 2 \rho(\mu-|\gamma|)+2 \rho|\gamma-\mu| \\
& \leq 4 \rho|\mu-\gamma| .
\end{aligned}
$$

If $\mu=0$, then both sides of the inequality are zero. In each case we have

$$
|\alpha-\beta|^{2} \leq 4 \rho|\mu-\gamma| \text {. }
$$

Let $\mathbf{g}_{1}, \mathbf{g}_{2}, \mathbf{g}_{3} \in \mathbf{C}, X_{1}=X \in H, X_{2}=X_{3}=Y \in H$. Since $F$ is p.d., the matrix $\mathrm{A}=\left(\left\langle F\left(\mathbf{g}_{i}-\mathbf{g}_{j}\right) X_{i}, X_{j}\right\rangle\right)_{i, j=1}^{3}$ is nonnegative. Thus (2) yields

$$
\begin{aligned}
& \left|\left\langle F\left(\mathbf{g}_{1}-\mathbf{g}_{2}\right) X, Y\right\rangle-\left\langle F\left(\mathbf{g}_{1}-\mathbf{g}_{3}\right) X, Y\right\rangle\right|^{2} \\
& \quad=4\langle F(\mathbf{0}) X, X\rangle\left|\langle F(\mathbf{0}) Y, Y\rangle-\left\langle F\left(\mathbf{g}_{2}-\mathbf{g}_{3}\right) Y, Y\right\rangle\right| .
\end{aligned}
$$


Putting $A=F\left(\mathbf{g}_{2}-\mathbf{g}_{1}\right)-F\left(\mathbf{g}_{3}-\mathbf{g}_{1}\right)$ and $X=A Y$ we obtain from (3)

$$
\|A Y\|^{4}=\left\langle A^{*} A Y, Y\right\rangle^{2} \leq 4\|A\|^{2}\|F(\mathbf{0})\|\|Y\|^{2} \mid\left\langle\left(F(\mathbf{0})-F\left(\mathbf{g}_{2}-\mathbf{g}_{3}\right)\right) Y, Y\right\rangle .
$$

Since $\|A\| \leq 2\|F(0)\|$ by Lemma 1 , (ii), we have

$$
\left\|\left(F\left(\mathbf{g}_{2}-\mathbf{g}_{1}\right)-F\left(\mathbf{g}_{3}-\mathbf{g}_{1}\right)\right) Y\right\|^{4} \leq 16\|F(\mathbf{0})\|^{3}\|Y\|^{2}\left|\left\langle\left(F(\mathbf{0})-F\left(\mathbf{g}_{2}-\mathbf{g}_{3}\right)\right) Y, Y\right\rangle\right|,
$$

which yields our assertion.

Definition 2. A p.d. function $F: 2 \mathrm{C} \rightarrow B(H)$ is called a continuous positive definite (c.p.d.) function, if it is weakly continuous at $\mathbf{0}$.

Note that Lemma 2 justifies the notation in Definition 2, since for p.d. functions weak continuity at $\mathbf{0}$, weak continuity, strong continuity, and even ultrastrong continuity coincide.

Lemma 3. Suppose that $G_{0}$ is a dense subgroup of the abelian topological group $G$ and that $D$ is a dense linear subset of $H$. If the mapping $F_{0}: 2 C_{0}=S_{N}(2 a) \times$ $\mathbf{R}^{M} \times G_{0} \rightarrow B(H)$ is such that the function $\left(\mathbf{g} \mapsto\left\langle F_{0}(\mathbf{g}) X, X\right\rangle\right)$ is continuous at 0 for all $X \in D$ and if (1) is satisfied with $F_{0}$ in place of $F$, then there is $a$ unique c.p.d. extension $F$ of $F_{0}$ to $2 \mathrm{C}$.

Proof. By Lemma 1, (ii), $F_{0}$ is p.d. and the image of $F_{0}$ is uniformly bounded. Hence the continuity assumption implies in fact weak continuity of $F_{0}$ at 0 . By Lemma 2, $F_{0}$ is uniformly weakly continuous. Since the weak closure of the image of $F_{0}$ is weakly compact and hence weakly complete, there is a weakly continuous extension $F$ of $F_{0}$ to $2 \mathrm{C}$. It is clear that this extension is unique and p.d.

\section{Positive Definite MatriX-VAlued distributions}

We start with the introduction of certain matrices of test functions. For $\varphi \in \mathscr{D}\left(\mathbf{R}^{N+M}\right)$, we define $\varphi^{*}$ by $\varphi^{*}(x, y)=\overline{\varphi(-x,-y)}, x \in \mathbf{R}^{N}, y \in \mathbf{R}^{M}$. Consider matrices $\Phi=\left(\varphi_{k l}\right)_{k, l \in N}$ and $\Psi=\left(\psi_{k l}\right)_{k, l \in N}$ in $M\left(\omega ; \mathscr{D}\left(\mathbf{R}^{N+M}\right)\right)$. We define an involution in $M\left(\omega ; \mathscr{D}\left(\mathbf{R}^{N+M}\right)\right)$ by $\Phi^{*}=\left(\xi_{k l}\right)_{k, l \in N}, \xi_{k l}=\varphi_{l k}^{*}$. A convolution in $M\left(\omega ; \mathscr{D}\left(\mathbf{R}^{N+M}\right)\right)$ is defined by $\Phi * \Psi=\left(\eta_{k l}\right)_{k, l \in \mathbf{N}}, \eta_{k l}=$ $\sum_{j \in N} \varphi_{k j} * \psi_{j l}$, where $*$ is the latter sum denotes the usual convolution. Let $M\left(\omega ; \mathscr{D}_{2 a}\right)_{+}$denote the cone in $M\left(\omega ; \mathscr{D}_{2 a}\right)$, which is generated by all elements of the form $\Phi^{*} * \Phi, \Phi \in M\left(\omega ; \mathscr{D}_{a}\right)$. Each element $\mathscr{F}=\left(f_{k l}\right)_{k, l \in N} \in$ $M\left(\infty ; \mathscr{D}_{2 a}^{\prime}\right)$ defines a linear functional on $M\left(\omega ; \mathscr{D}_{2 a}\right)$ by

$$
\mathscr{F}(\Phi)=\sum_{k, l \in N} f_{k l}\left(\varphi_{l k}\right)
$$

We assume that $M\left(\infty ; \mathscr{D}_{2 a}^{\prime}\right)$ carries the appropriate weak topology. Note that the latter is Hausdorff. We say that $\mathscr{F}$ is positive $\left(\mathscr{F} \in M\left(\infty ; \mathscr{D}_{2 a}^{\prime}\right)_{+}\right)$iff $\mathscr{F}(\Phi) \geq 0$ for $\Phi \in M\left(\omega ; \mathscr{D}_{2 a}\right)_{+}$. It is easy to see that $\mathscr{F}$ is positive iff $\sum_{k, l \in N} f_{k l}\left(\varphi_{k} * \varphi_{l}^{*}\right) \geq 0$ for all finite sequences (i.e., all but finitely many $\varphi_{k}$ 
are zero) $\left(\varphi_{k}\right)_{k \in N}$. Writing elements of $M\left(\infty ; \mathscr{D}_{2 a}^{\prime}\right) \otimes M(n)$ as block matrices, we may identify the latter space with $M\left(\infty ; \mathscr{D}_{2 a}^{\prime}\right)$. Thus $M\left(\infty ; \mathscr{D}_{2 a}^{\prime}\right) \otimes M(n)$ inherits the order structure from $M\left(\infty ; \mathscr{D}_{2 a}^{\prime}\right)$ and we may speak about positive elements in the tensor product. In more detail, $\left(\left(f_{k l, i j}\right)_{k, l \in \mathbb{N}}\right)_{i, j=1}^{n} \in$ $M\left(\infty ; \mathscr{D}_{2 a}^{\prime}\right) \otimes M(n)$ is positive, if $\sum_{k, l \in \mathrm{N}} \sum_{i, j=1}^{n} f_{k l, i j}\left(\varphi_{i k} * \varphi_{j l}^{*}\right) \geq 0$ for all finite sequences $\left(\left(\varphi_{i k}\right)_{k \in N}\right)_{i=1}^{n}$ in $\mathscr{D}_{a}$.

If now $H$ is a separable Hilbert space, $\left(\mathbf{e}_{n}\right)_{n \in N}$ an orthonormal basis in $H$, and if $F: 2 \mathrm{C} \rightarrow B(H)$ is weakly continuous, we associate a continuous mapping

$$
\mathscr{F}_{F}: G \ni g \mapsto\left(f_{k l, g}\right)_{k, l \in N} \in M\left(\infty ; \mathscr{D}_{2 a}^{\prime}\right)
$$

to $F$ by

$$
f_{k l, g}(\varphi):=\int_{S_{N}(2 a) \times \mathbf{R}^{M}}\left\langle F(x, y, g) \mathbf{e}_{k}, \mathbf{e}_{l}\right\rangle \varphi(x, y) d x d y, \quad \varphi \in \mathscr{D}_{2 a} .
$$

Lemma 4. $F$ is p.d. iff $\left(\mathscr{F}_{F}\left(g_{i}-g_{j}\right)\right)_{i, j=1}^{n}$ is positive in $M\left(\infty ; \mathscr{D}_{2 a}^{\prime}\right) \otimes M(n)$ for all $g_{1}, \ldots, g_{n} \in G, n \in \mathbb{N}$.

Proof. Let $n, m \in \mathbb{N}, \varphi_{11}, \ldots, \varphi_{n m} \in \mathscr{D}_{a}$, and $g_{1}, \ldots, g_{n} \in G$. Then the following equality holds.

$$
\begin{aligned}
& \sum_{i, j=1}^{n} \sum_{k, l=1}^{m} f_{k l, g_{i}-g_{j}}\left(\varphi_{i k} * \varphi_{j l}^{*}\right) \\
& =\sum_{i, j=1}^{n} \sum_{k, l=1}^{m} \int_{S_{i}(2 a) \times \mathbf{R}^{y}} \int_{\mathbf{R}^{N+. u}}\left\langle F\left(x, y, g_{i}-g_{j}\right) \mathbf{e}_{k}, \mathbf{e}_{1}\right\rangle \\
& \times \varphi_{i k}\left(x-x^{\prime}, y-y^{\prime}\right) \overline{\varphi_{j l}\left(-x^{\prime},-y^{\prime}\right)} d x^{\prime} d y^{\prime} d x d y \\
& =\sum_{i, j=1}^{n} \sum_{k, l=1}^{m} \int_{S_{N}(a) \times \mathbf{R}^{M}} \int_{S_{i}(a) \times \mathbf{R}^{M}}\left\langle F\left(x-x^{\prime}, y-y^{\prime}, g_{i}-g_{j}\right) \mathbf{e}_{k}, \mathbf{e}_{1}\right\rangle \\
& \times \varphi_{i k}(x, y) \overline{\varphi_{j l}\left(x^{\prime}, y^{\prime}\right)} d x^{\prime} d y^{\prime} d x d y \\
& =\sum_{i, j=1}^{n} \int_{S_{N}(a) \times \mathbf{R}^{M}} \int_{S_{N}(a) \times \mathbf{R}^{M}}\left\langle F\left(x-x^{\prime}, y-y^{\prime}, g_{i}-g_{j}\right)\right. \\
& \times \sum_{k=1}^{m} \varphi_{i k}(x, y) \mathbf{e}_{k} \\
& \left.\sum_{l=1}^{m} \varphi_{j l}\left(x^{\prime}, y^{\prime}\right) \mathbf{e}_{1}\right\rangle d x^{\prime} d y^{\prime} d x d y .
\end{aligned}
$$

Suppose now that $F$ is p.d. Approximating the integrals on the right-hand side of (4) by suitable (e.g. equidistant) Riemann sums, we obtain its nonnegativity. Thus $\left(F_{F}\left(g_{i}-g_{j}\right)\right)_{i, j=1}^{n}$ is positive in $M\left(\infty ; \mathscr{D}_{2 a}^{\prime}\right) \otimes M(n)$. 
Assume now that the matrix $\left(\mathscr{F}_{F}\left(g_{i}-g_{j}\right)\right)_{i, j=1}^{n}$ is positive in the tensor product. Let $X_{1}, \ldots, X_{n} \in D:=1 . h .\left\{\mathbf{e}_{k} ; k \in \mathbb{N}\right\}$. Fix $m \in \mathbb{N}$ such that $\alpha_{i k}:=\left\langle X_{i}, \mathbf{e}_{k}\right\rangle$ vanishes for $k>m$ and all $i=1, \ldots, n$. Now let $\varphi_{i k}$ run through a sequence of test functions converging (as measures) weakly to the point measure at $\left(x_{i}, y_{i}\right)$ with mass $\alpha_{i k}$. Since the term remains nonnegative and converges to $\sum_{i, j=1}^{n}\left\langle F\left(\mathbf{g}_{i}-\mathbf{g}_{j}\right) X_{i}, X_{j}\right\rangle, \mathbf{g}_{i}=\left(x_{i}, y_{i}, g_{i}\right)$, we see that (1) is satisfied with $\mathbf{C}_{0}=\mathbf{C}$. By Lemma $1(\mathrm{ii}), F$ is p.d.

Lemma 4 motivates the introduction of matrix-valued distributions as generalizations of operator-valued functions in the following definition. The notion depends on the decomposition of $\mathbf{G}$ as a direct product of course. Since there is no danger of confusion, we will not express this dependence in notation.

Definition 3. By a $G$-continuous matrix-valued distribution on $2 \mathrm{C}$ we mean a continuous mapping $\mathscr{F}: G \rightarrow M\left(\infty ; \mathscr{D}_{2 a}^{\prime}\right)$. It is called $N$-radial, if each entry of $\mathscr{F}(g)$ is $N$-radial. The matrix-valued distribution $\mathscr{F}$ is called positive definite, if $\left(\mathscr{F}\left(g_{i}-g_{j}\right)\right)_{i, j=1}^{n}$ is positive in $M\left(\infty ; \mathscr{D}_{2 a}^{\prime}\right) \otimes M(n)$ for all $g_{1}, \ldots, g_{n} \in G, n \in \mathbb{N}$.

I.e., $\mathscr{F}$ is p.d. iff for all $n, m \in \mathbb{N}, g_{1}, \ldots, g_{n} \in G$, and $\varphi_{11}, \ldots, \varphi_{n m} \in \mathscr{D}_{a}$ the expression $\sum_{i, j=1}^{n} \sum_{k, l=1}^{m} f_{k l, g_{i}-g_{j}}\left(\varphi_{i k} * \varphi_{j l}^{*}\right)$ is nonnegative.

\section{NUCLEAR AUXILARY SPACES}

In this chapter we construct certain nuclear spaces, which provide a basis for later applications of the nuclear spectral theorem. For general properties of nuclear spaces we refer to [19]. Some of the following facts were proved in a similar form in [17] already.

Suppose that $G_{0}$ is a countable abelian group. In later applications $G_{0}$ will be a dense subgroup of an abelian separable topological group $G$.

Let

$$
E_{a}=E\left(a ; N, M, G_{0}\right)=\bigoplus_{k \in \mathbf{N}, g \in G_{0}} E_{k, g}
$$

be the topological direct sum of vector spaces $E_{k, g} \cong \mathscr{D}_{a}$. Let $J_{k g}: \mathscr{D}_{a} \rightarrow$ $J_{k g}\left(\mathscr{D}_{a}\right)=E_{k, g} \subseteq E_{a}$ denote the canonical injection. Similarly we define a vector space

$$
\mathscr{E}_{a}=\mathscr{E}\left(a ; N, M, G_{0}\right)=\bigoplus_{k, l \in N, g \in G_{0}} \mathscr{E}_{k l, g},
$$

where $\mathscr{E}_{k l, g}$ is topologically and algebraically isomorphic to $\mathscr{D}_{2 a}$. Let $\mathscr{J}_{k l g}: \mathscr{D}_{2 a}$ $\rightarrow \mathscr{E}_{k l, g} \subseteq \mathscr{E}_{a}$ denote the appropriate canonical injection. Informally speaking, $E_{a}$ and $\mathscr{E}_{a}$ are spaces of sequences and of matrices of test functions, which additionally depend on the parameter of $g \in G_{0}$.

We introduce an involution in $E_{a}$ and a convolution $*: E_{a} \times E_{a} \rightarrow \mathscr{E}_{a}$ by

$$
\left(J_{k g} \varphi\right)^{*}=\left(J_{k,-g} \varphi^{*}\right), \quad\left(J_{k g} \varphi\right) *\left(J_{l h} \psi\right)=\mathscr{J}_{k l, g+h}(\varphi * \psi),
$$

$k, l \in \mathbb{N}, g, h \in G_{0}, \varphi, \psi \in \mathscr{D}_{a}$, and by linear extension of these relations to the whole spaces. 
Lemma 5. Involution and convolution defined above are continuous.

Proof. The continuity of the involution is obvious. To show the continuity of the convolution it is sufficient to prove the continuity of the restricted mappings *: $J_{k g}\left(\mathscr{D}_{a}\right) \times J_{l h}\left(\mathscr{D}_{a}\right) \rightarrow \mathscr{J}_{k l, g+h}\left(\mathscr{D}_{2 a}\right) \subseteq \mathscr{E}_{a}$ (cf. [6, 7.7.11]). Hence we have to prove the continuity of the usual convolution $*: \mathscr{D}_{a} \times \mathscr{D}_{a} \rightarrow \mathscr{D}_{2 a}$. Since $\mathscr{D}_{a}$ is a $D F$-space (cf. [11, $\S 40,2]$ ), it suffices to show that the convolution is separately continuous. The latter follows from a straightforward calculation using seminorms.

Let $\mathscr{D}_{a}^{\#}$ denote the subspace of all $N$-radial test functions in $\mathscr{D}_{a}$. Moreover, $E_{a}^{\#}:=1 . h .\left\{J_{k g}(\varphi) ; \varphi \in \mathscr{D}_{a}^{\#}, k \in \mathbb{N}, g \in G_{0}\right\}$ is a linear subspace of $E_{a}$.

Lemma 6. $E_{a}$ and $E_{a}^{\#}$ are separable nuclear spaces.

Proof. Both spaces are countable direct sums of separable nuclear spaces $\mathscr{D}_{a}$ resp. $\mathscr{D}_{a}^{\#}$. Thus they are also of that kind. (cf. $[19,5.2 .2]$ ).

Lemma 7. The set $\left\{\varphi * \psi ; \varphi, \psi \in \mathscr{D}_{a}^{\#}\right\}$ is total in $\mathscr{D}_{2 a}^{\#}$.

Proof. Functions of the form $\varphi \otimes \psi, \varphi \in \mathscr{D}\left(S_{N}(a)\right), \psi \in \mathscr{D}\left(\mathbb{R}^{N}\right)$, span a dense subspace of $\mathscr{D}_{a}$. Since the mapping $\varphi \otimes \psi \mapsto(\varphi \otimes \psi)^{\#}=\varphi^{\#} \otimes \psi$, is continuous, elements of the form $\varphi \otimes \psi, \varphi \in \mathscr{D}\left(S_{N}(a)\right)^{\#}:=\left\{\varphi \in \mathscr{D}\left(S_{N}(a)\right)\right.$; $\varphi$ radial $\}, \psi \in \mathscr{D}\left(\mathbf{R}^{M}\right)$, are total in $\mathscr{D}_{a}^{\#}$. Because of $\left(\varphi_{1} \otimes \psi_{1}\right) *\left(\varphi_{2} \otimes \psi_{2}\right)=$ $\left(\varphi_{1} * \varphi_{2}\right) \otimes\left(\psi_{1} * \psi_{2}\right)$ it is sufficient to prove that $\left\{\varphi_{1} * \varphi_{2} ; \varphi_{1}, \varphi_{2} \in \mathscr{D}\left(S_{N}(a)\right)^{\#}\right\}$ is total in $\mathscr{D}\left(S_{N}(2 a)\right)^{\#}$ and that $\left\{\psi_{1} * \psi_{2} ; \psi_{1}, \psi_{2} \in \mathscr{D}\left(\mathbb{R}^{M}\right)\right\}$ is total in $\mathscr{D}\left(\mathbb{R}^{M}\right)$. The first assertion is Lemma 6.1 in [17] and the latter is clear.

Lemma 8. Every element of $\mathscr{D}_{a}^{\# \prime}$ is the restriction to $\mathscr{D}_{a}^{\#}$ of a unique $N$-radial distribution of $\mathscr{D}_{a}^{\prime}$.

Proof. The proof is quite similar to the case where $M=0$, which was carried out in [17, Proposition 1].

For $y \in \mathbb{R}^{M}$ and $g \in G_{0}$, we define a continuous linear operator $V(y, g)$ in $E_{a}$ by $V(y, g) J_{k, h} \varphi=J_{k, g+h} \psi$, where $\psi$ is defined by

$$
\psi\left(x^{\prime}, y^{\prime}\right)=\varphi\left(x^{\prime}, y^{\prime}-y\right), \quad \varphi \in \mathscr{D}_{a}, x^{\prime} \in \mathbb{R}^{N}, y^{\prime} \in \mathbb{R}^{M} \text {. }
$$

The mapping $(y, g) \mapsto V(y, g)$ defines a representation of $\mathbf{R}^{M} \times G_{0}$ in $E_{a}$. Later on we will use $V$ to define a strongly continuous unitary representation of $\mathbb{R}^{M} \times G$ in a certain Hilbert space, where $G$ is a locally compact abelian group containing $G_{0}$ as a dense subgroup. The appropriate Hilbert space will be obtained by GNS-construction. To prepare this, we have to consider suitable positive functionals $T$ of $\mathscr{E}_{a}^{\prime}$.

Definition 4. $T \in \mathscr{E}_{a}^{\prime}$ is called positive, if $T\left(\Phi * \Phi^{*}\right) \geq 0$ for all $\Phi \in E_{a}$.

The connection between positive definite $G$-continuous matrix-valued distributions and positive functionals is as follows. For a given $G$-continuous 
matrix-valued distribution $\mathscr{F}$ on $2 \mathrm{C}$ and a fixed countable dense subgroup $G_{0}$ of $G$ we define a functional $T_{\mathscr{F}} \in \mathscr{E}_{a}^{\prime}$ by $T_{\mathscr{F}}\left(\mathscr{L}_{k l, g} \varphi\right)=f_{k l, g}(\varphi), k, l \in \mathbb{N}$, $g \in G_{0}, \varphi \in \mathscr{D}_{a}$.

Lemma 9. $T_{\mathscr{F}}$ is positive if and only if $\mathscr{F}=\left(f_{k l}\right)_{k, l \in \mathrm{N}}$ is p.d.

Proof. Consider $\Phi \in E_{a}, \Phi=\sum_{g \in G_{0}} \sum_{k=1}^{\infty} J_{k, g} \varphi_{k g}, \varphi_{k g} \in \mathscr{D}_{a} . \varphi_{k g} \not \equiv 0$ only for finitely many $g$ 's, say $g_{1}, \ldots, g_{n}$. Then

$$
\begin{aligned}
T_{\mathscr{F}}\left(\Phi * \Phi^{*}\right) & =T_{\mathscr{F}}\left(\sum_{k, l=1}^{\infty} \sum_{i, j=1}^{n} \mathscr{J}_{k l, g_{i}-g_{j}}\left(\varphi_{k g_{i}} * \varphi_{l g_{j}}^{*}\right)\right) \\
& =\sum_{k, l=1}^{\infty} \sum_{i, j=1}^{n} f_{k l, g_{i}-g_{j}}\left(\varphi_{k g_{i}} * \varphi_{l g_{j}}^{*}\right) .
\end{aligned}
$$

Now the assertion is easy, since $G_{0}$ is dense in $G$ and $\mathscr{F}$ is $G$-continuous.

In the following we want to show that $G$-continuous matrix-valued positive definite distributions and continuous operator-valued positive definite functions possess a certain integral representation if $N=1$ or if they are $N$-radial. This will follow from the investigation of the appropriate functionals in $\mathscr{E}_{a}^{\prime}$. This integral representation will be obtained from a suitable direct integral Hilbert space and by applying the nuclear spectral theorem. The appropriate concepts (in a form which applies to our situation) will be developed in the next three paragraphs.

\section{THE NUCLEAR SPECTRAL THEOREM}

Theorems of the following kind are well known (cf. [8, 16, 2, 17]). Here we give a special formulation, which will be proved for completeness. For the concept of direct integrals we refer to [5].

Proposition 10. Suppose that $E$ is a nuclear space and $H=\int_{\Lambda}^{\oplus} H(\lambda) d \nu(\lambda) a$ separable direct integral Hilbert space. If $J: E \rightarrow H$ is a continuous linear mapping, then there are continuous linear mappings $J_{\lambda}: E \rightarrow H(\lambda)$ for all $\lambda \in$ $\Lambda$ such that for each $e \in E$ the vector field $\left(J_{\lambda} e ; \lambda \in \Lambda\right)$ belongs to $H$ and $(J e)(\lambda)=J_{\lambda} e$-a.e. on $\Lambda$.

Proof. Since $J$ is continuous, there is an equicontinuous sequence $\left(e_{n}^{\prime}\right)_{n \in N}$ of linear functionals on $E$ (i.e. there is a continuous seminorm $p$ on $E$ such that $\left|e_{n}^{\prime}(e)\right| \leq p(e)$ for all $e \in E$ and $\left.n \in \mathbb{N}\right)$, a bounded sequence $\left(X_{n}\right)_{n \in \mathbb{N}}$ of vectors in $H$, and a sequence $\left(\sigma_{n}\right)_{n \in N}$ of nonnegative numbers belonging to $l_{1}$ (i.e. $\left.\sum_{n \in \mathrm{N}} \sigma_{n}<\infty\right)$ such that $J e=\sum_{n=1}^{\infty} \sigma_{n} e_{n}^{\prime}(e) X_{n}$ for all $e \in E$. Suppose that $X_{n}=\int_{\Lambda}^{\oplus} X_{n}(\lambda) d \nu(\lambda)$. Now

$$
\begin{aligned}
\sum_{n=1}^{\infty} \sigma_{n}\left\|X_{n}\right\|^{2} & =\sum_{n=1}^{\infty} \int_{\Lambda} \sigma_{n}\left\|X_{n}(\lambda)\right\|_{\lambda}^{2} d \nu(\lambda) \\
& =\int_{\Lambda} \sum_{n=1} \sigma_{n}\left\|X_{n}(\lambda)\right\|_{\lambda}^{2} d \nu(\lambda)<\infty
\end{aligned}
$$


Thus there is a $\nu$-null set $\mathscr{N}$ such that $\sum_{n=1}^{\infty} \sigma_{n}\left\|X_{n}(\lambda)\right\|_{\lambda}^{2}<\infty$ for all $\lambda \in$ $\Lambda \backslash \mathscr{N}$. Without loss of generality we may assume that $X_{n}(\lambda)=0$ if $\lambda \in \mathscr{N}$. Thus $\sum_{n=1}^{\infty} \sigma_{n}\left\|X_{n}(\lambda)\right\|_{\lambda}^{2}<\infty$ for all $\lambda \in \Lambda$. (From now on, the vector fields $\left(X_{n}(\lambda) ; \lambda \in \Lambda\right)$ are fixed.) We define linear mappings $J_{\lambda}: E \rightarrow H(\lambda)$ by $J_{\lambda} e=$ $\sum_{n=1}^{\infty} \sigma_{n} e_{n}^{\prime}(e) X_{n}(\lambda), e \in E . J_{\lambda}$ is continuous. Indeed,

$$
\begin{aligned}
\left\|J_{\lambda} e\right\|_{\lambda} & \leq \sum_{n=1}^{\infty}\left|e_{n}^{\prime}(e)\right| \sigma_{n}^{1 / 2}\left\|\sigma_{n}^{1 / 2} X_{n}(\lambda)\right\|_{\lambda} \\
& \leq p(e)\left(\sum_{n=1}^{\infty} \sigma_{n}\right)^{1 / 2}\left(\sum_{n=1}^{\infty} \sigma_{n}\left\|X_{n}(\lambda)\right\|_{\lambda}^{2}\right)^{1 / 2}
\end{aligned}
$$

which shows the continuity of $J_{\lambda}$.

Let $e \in E$. We like to show that $(J e)(\lambda)=J_{\lambda} e \quad \nu$-a.e. Set

$$
S_{k} e=\sum_{n=1}^{k} \sigma_{n} e_{n}^{\prime}(e) X_{n}, \quad k \in \mathbb{N} .
$$

Since the sequence $\left(S_{k} e\right)_{k \in N}$ converges to $J e$ in $H$, there exists a subsequence $\left(S_{k_{m}} e\right)_{m \in N}$ such that $\left(\left(S_{k_{m}} e\right)(\lambda)\right)_{m \in N}$ converges to $(J e)(\lambda)$ in $H(\lambda)$ for $\nu$-a.a. $\lambda \in \Lambda$. [5, part II, Chapter 1, Proposition 5]. But, by the definition of $J_{\lambda}$, the sequence $\left(\left(S_{k_{m}} e\right)(\lambda)\right)_{m \in N}$ converges to $J_{\lambda} e$, which yields the assertion.

We want to conclude this paragraph with the description of how one obtains a suitable Hilbert space from a positive functional $T$ in $\mathscr{E}_{a}^{\prime}$. This construction is very similar to the well-known GNS-construction. Let $T$ denote a positive functional of $\mathscr{E}_{a}^{\prime}$. We define a semiscalar product on $E_{a}$ by $\langle\Phi, \Psi\rangle:=T\left(\Phi * \Psi^{*}\right)$, $\Phi, \Psi \in E_{a}$. By Lemma 5 this semiscalar product is simultaneously continuous on $E_{a}$. Therefore $N:=\left\{\Phi \in E_{a} ;\langle\Phi, \Phi\rangle=0\right\}$ is a closed subspace of $E_{a}$. Let $H$ denote the completion of $E_{a} / N$ w.r.t. the norm $(\Phi+N) \mapsto\|\Phi+N\|:=$ $(\langle\Phi, \Phi\rangle)^{1 / 2}$. Of course, $H$ is a Hilbert space. Let $J: E_{a} \rightarrow H$ denote the natural injection, i.e., $J \Phi=\Phi+N \in H, \Phi \in E_{a}$. By Lemma $5, J$ is a continuous linear mapping. Hence we may apply Proposition 10 provided that $H$ has a direct integral structure. Suppose we are given a $N$-radial positive element of $\mathscr{E}_{a}^{\prime}$. Then we use the nuclear space $E_{a}^{\#}$ to construct a Hilbert space $H^{\#}$ in a quite similar way.

In the following paragraphs we show that a suitable direct integral representation can be given in case of $N=1$ for a Hilbert space $\mathscr{H}$ containing $H$, and for $H^{\#}$ in the $N$-radial case.

\section{EXTENSIONS OF COMMUTING OPERATORS}

Similar assertions as in the following proposition were proved already in [10 and 22].

Proposition 11. Let $H$ denote a separable Hilbert space, $G$ a topological abelian group, $G_{0}$ a dense subgroup of $G$ and $A: D(A) \rightarrow H$ a symmetric operator. 
Suppose that $U: G \rightarrow B(H)$ is a strongly continuous representation of $G$, which strongly commutes with $A$ in the following sense: $U(g) D(A) \subseteq D(A)$ for all $g \in G_{0}$ and $U(g) A X=A U(g) X$ for all $g \in G_{0}$ and $X \in D(A)$. Then there are

(i) a separable Hilbert space $\mathscr{H}$ which contains $H$ as a closed subspace.

(ii) a selfadjoint extension $\mathscr{A}$ of $A$ in $\mathscr{H}$, and

(iii) a unitary strongly continuous representation $\mathscr{U}$ of $G$ in $\mathscr{H}$ which coincides with $U$ on $H$ such that $\mathscr{U}(g) D(\mathscr{A}) \subseteq D(\mathscr{A})$ for all $g \in G$ and $\mathscr{U}(g) \mathscr{X}=\mathscr{M}(g) \mathscr{X}$ for all $g \in G$ and $\mathscr{X} \in D(\mathscr{A})$. If $A$ is nonnegative, we may choose $\mathscr{H}=H$ (thus $\mathscr{U}=U$ ) and $\mathscr{A}$ as the Friedrichs extension of $A$.

Proof. 1. Since $A$ is a symmetric and hence closable, $\bar{A}$ commutes strongly with $U(g), g \in G_{0}$, i.e. $U(g) \bar{A} \subseteq \bar{A} U(g)$.

2. $\bar{A}$ commutes strongly with $U(g), g \in G$. This follows from the continuity of $U$ and the closedness of $\bar{A}$.

3. Let $\mathscr{H}:=H \oplus H, A_{1}:=\bar{A} \oplus(-\bar{A})$ (i.e. especially $D\left(A_{1}\right)=D(\bar{A}) \oplus D(\bar{A})$ ), and $\mathscr{U}$ be given by $\mathscr{U}(g)=U(g) \oplus U(g), g \in G$. It is easy to see that $A_{1}$ is a densely defined closed symmetric operator which strongly commutes with $\mathscr{U}(g), g \in G$. Consider the deficiency spaces $E_{ \pm}=\left\{\mathscr{Z} \in D\left(A_{1}^{*}\right) ; A_{1}^{*} \mathscr{Z}=\right.$ $\pm i \mathscr{X}\}$ and the Cayley transform $C_{1}:\left(A_{1}+i\right) D\left(A_{1}\right) \ni\left(A_{1}+i\right) \mathscr{X} \mapsto\left(A_{1}-i\right) \mathscr{X}$ $\in\left(A_{1}-i\right) D\left(A_{1}\right)$ of $A_{1}$. We have $\left(\left(A_{1} \pm i\right) D\left(A_{1}\right)\right)^{\perp}=\{(X, Y) \in H \oplus H$; $\langle X,(\bar{A} \pm i) z\rangle=0$ and $\langle Y,(\bar{A} \mp i) Z\rangle=0$ for all $Z \in D(\bar{A})\}=E_{\mp} \oplus E_{ \pm}$. Thus we may define a unitary operator $\mathscr{C}$ in $\mathscr{H}$ by $\mathscr{C} \mid\left(A_{1}+i\right) D\left(A_{1}\right)=C_{1}$ and $\mathscr{C}(X, Y)=(Y, X)$ for $X \in E_{+}$and $Y \in E_{-}$. Hence $\mathscr{C}$ is a unitary operator mapping $\mathscr{H}$ onto $\mathscr{H}$.

4. We show that $\operatorname{ker}(\mathscr{C}-1)=\{0\}$. As it was shown in 3., we may write an arbitrary vector $\mathscr{Z}$ of $\mathscr{C}$ as follows $\left(X_{1}+(\bar{A}+i) X_{2}, Y_{1}+(-\bar{A}+i) Y_{2}\right), X_{1} \in E_{+}$, $Y_{1} \in E_{-}, X_{2}, Y_{2} \in D(\bar{A})$. Suppose that it belongs to $\operatorname{ker}(\mathscr{C}-1)$. Regarding the first component of the resulting vector, we obtain $Y_{1}+(\bar{A}-i) X_{2}-X_{1}-(\bar{A}+i) X_{2}=$ $Y_{1}-X_{1}-2 i X_{2}=0$, which implies $X_{1}=X_{2}=Y_{1}=0$, since $E_{+}, E_{-}$, and $D(\bar{A})$ are linearly independent. (These spaces are even orthogonal w.r.t. the graph topology of $A^{*}$, as it is well known from the extension theory of symmetric operators.) Similarly, $Y_{2}=0$. Thus $\operatorname{ker}(\mathscr{C}-1)=\{0\}$ and we may define a symmetric operator $\mathscr{A}$ by $D(\mathscr{A})=(\mathscr{C}-1) \mathscr{H}$ and $\mathscr{A}(\mathscr{C}-1) \mathscr{C}=$ $-i(\mathscr{C}+1) \mathscr{X}, \mathscr{X} \in \mathscr{H}$. Note that $D(\mathscr{A})$ is dense, since

$$
\overline{(\mathscr{C}-1) \mathscr{H}}=\left(\operatorname{ker}\left(\mathscr{C}^{*}-1\right)\right)^{\perp}=\left(\mathscr{C}^{*} \operatorname{ker}(\mathscr{C}-1)\right)^{\perp}=\mathscr{H} .
$$

It is easy to see that $\mathscr{A}$ is an extension of $A_{1}$ and hence of $A$. Moreover, $\mathscr{A}$ is selfadjoint, since its Cayley transform $\mathscr{C}$ is unitary. 
5. $\mathscr{A}$ and $\mathscr{U}(g), g \in G$, commute strongly, Indeed, consider an arbitrary vector $\mathscr{Z}$ of $\mathscr{H}$. Suppose that it is written as in 4 . Then

$$
\begin{aligned}
\mathscr{U}(g) \mathscr{E} \mathscr{Z} & =\mathscr{U}(g)\left(Y_{1}+(\bar{A}-i) X_{2}, X_{1}+(-\bar{A}-i) Y_{2}\right) \\
& =\left(U(g) Y_{1}+(\bar{A}-i) U(g) X_{2}, U(g) X_{1}+(-\bar{A}-i) U(g) Y_{2}\right) \\
& =\mathscr{C} U(g) \mathscr{Z},
\end{aligned}
$$

since $U(g)$ strongly commutes with $\bar{A}$. i.e. $\mathscr{U}(g), g \in G$, and $\mathscr{C}$ commute, which implies that $\mathscr{U}(g)$ and $\mathscr{A}$ commute strongly. This completes the proof of the first part.

6. Suppose now that $A$ is nonnegative. Then we obtain that $\bar{A}$ is nonnegative and commutes strongly with all $U(g), g \in G$, according to 2. Consider the *-algebra $W=1$.h. $\{U(g) ; g \in G\}$. Consider a net $\left(B_{\lambda}\right)_{\lambda \in \Lambda}$ in $W$, which strongly converges to some $B \in B(H)$. Since $B_{\lambda} D(\bar{A}) \subseteq D(\bar{A})$ and $\bar{A} B_{\lambda} X=B_{\lambda} \bar{A} X$ for all $\lambda \in \Lambda$ and $X \in D(\bar{A})$, we have $B_{\lambda} X \rightarrow B X$ and $\bar{A} B_{\lambda} X=$ $B_{\lambda} A X \rightarrow B A X$. Since $\bar{A}$ is closed, $B X \in D(\bar{A})$ and $\bar{A} B X=B \bar{A} X$. Thus all operators belonging to the von Neumann algebra $W^{\prime \prime}$ commute strongly with $\bar{A}$. Fix any projection $P \in W^{\prime \prime}$. Then $H$ can be written as orthogonal direct sum $(1-P) H \oplus P H$. Since $\bar{A}$ commutes strongly with $P$, we have $\bar{A}=A_{1} \oplus A_{2}$, $A_{1}=(1-P) \bar{A} \uparrow(1-P) D(\bar{A}), A_{2}=P \bar{A} \uparrow P D(\bar{A})$. It is clear from the construction of the Friedrichs extensions $\bar{A}_{F}=A_{F}, A_{1 F}$, and $A_{2 F}$ of $A, A_{1}$, and $A_{2}$, respectively, that $A_{F}=A_{1 F} \oplus A_{2 F}$. Especially, $A_{F}$ commutes strongly with $P$. Since $W^{\prime \prime}$ is the strong closure of the linear hull of all of its projections, $A_{F}$ commutes with all operators of $W^{\prime \prime}$ in the strong sense. Especially $A_{F}$ commutes strongly with all $U(g), g \in G$, which completes the proof.

Note that the extension of $A$ in Proposition 11 is not unique in general.

\section{Direct integral Hilbert Spaces}

Definition 5 (cf. [1]). Let $\mathscr{Z}$ denote a locally compact topological space and $H$ a Hilbert space. A projection-valued measure $P$ on $\mathscr{Z}$ is a mapping which assigns to each Borel subset $\Delta$ of $\mathscr{Z}$ a projection $P(\Delta)$ such that the following conditions are satisfied:

(i) $P(\varnothing)=0$ and $P(\mathscr{X})$ is the identity of $H$.

(ii) $P\left(\Delta_{1}\right) P\left(\Delta_{2}\right)=P\left(\Delta_{1} \cap \Delta_{2}\right)$ for all Borel sets $\Delta_{1}, \Delta_{2}$.

(iii) $P$ is $\sigma$-additive w.r.t. the weak operator topology.

(iv) $P$ is regular, i.e., $P(\Delta)=\inf \{P(\Omega) ; \Omega \subseteq \mathscr{X}$ open, $\Delta \subseteq \Omega\}$.

Proposition 12. Suppose that $U$ is a strongly continuous unitary representation of a locally compact abelian group $G$ in a separable Hilbert space $H$ and that $A$ is a selfadjoint operator in $H$, which strongly commutes with $U(g), g \in G$. Then there are a finite regular measure $\mu$ on $\mathbb{R} \times G^{*}$, and a family $H(\tau, \gamma)$, $\tau \in \mathbb{R}, \gamma \in G^{*}$, of Hilbert spaces such that

(i) $H=\int_{\mathbf{R} \times G^{*}}^{\oplus} H(\tau, \gamma) d \mu(\tau, \gamma)$, 
(ii) $U(g) X=\int_{\mathbf{R} \times G^{*}}^{\oplus}[\gamma, g] \times(\tau, \gamma) d \mu(\tau, \gamma)$, for $X=\int_{\mathbf{R} \times G^{*}}^{\oplus} X(\tau, \gamma) d \mu(\tau, \gamma) \in$ $H, g \in G$, and

(iii) $A X=\int_{\mathbf{R} \times G^{*}}^{\oplus} \tau X(\tau, \gamma) d \mu(\tau, \gamma)$ for $X=\int_{\mathbf{R} \times G^{*}}^{\oplus} X(\tau, \gamma) d \mu(\tau, \gamma) \in D(A)$. If $A$ is nonnegative, we have $\mu\left(\left(\mathbb{R} \backslash \mathbf{R}_{+}\right) \times G^{*}\right)=0$.

Proof. The one-parametric group $t \mapsto \exp (-i t A)$ commutes with $U(g)$, since $A$ strongly commutes with $U(g), g \in G$. Thus $(t, g) \mapsto \exp (-i t A) U(g)$ defines a strongly continuous unitary representation of $\mathbb{R} \times G$. According to the SNAG-Theorem (cf. e.g. [1]) there is a projection-valued measure $P$ on $\mathrm{R} \times G^{*}$ such that

$$
\langle\exp (-i t A) U(g) X, Y\rangle=\int_{\mathbf{R} \times G^{*}} \exp (-i t \tau)[\gamma, g]\langle d P(\tau, \gamma) X, Y\rangle,
$$

$t \in \mathbf{R}, g \in G, X, Y \in H$. For $g=0$ and $X \in D(A)$, we differentiate both sides and obtain

$$
\langle A X, Y\rangle=\int_{\mathbf{R} \times G^{*}} \tau\langle d P(\tau, \gamma) X, Y\rangle, \quad x \in D(A), Y \in H .
$$

For $t=0$, we obtain

$$
\langle U(g) X, Y\rangle=\int_{\mathbf{R} \times G^{*}}[\gamma, g]\langle d P(\tau, \gamma) X, Y\rangle, \quad g \in G, X, Y \in H .
$$

Following [23] there is a finite regular Borel measure $\mu$ on $\mathrm{R} \times G^{*}$ and a field of Hilbert spaces $H(\tau, \gamma), \tau \in \mathbf{R}, \gamma \in G^{*}$, such that

$$
H=\int_{\mathbf{R} \times G^{*}}^{\oplus} H(\tau, \gamma) d \mu(\tau, \gamma) \text { and } P(\Delta)=\int_{\mathbf{R} \times G^{*}}^{\oplus} \chi_{\Delta}(\tau, \gamma) 1_{(\tau, \gamma)} d \mu(\tau, \gamma),
$$

where $\chi_{\Delta}$ denotes the characteristic function of the Borel set $\Delta$ and $1_{(\tau, \gamma)}$ the identity in $H(\tau, \gamma)$. Now the assertions (ii) and (iii) follow from (5) and (6).

\section{Positive DEFINITE DISTRIBUTIONS ON A STRIP}

Recall that we fixed a decomposition $\mathbb{R}^{N+M} \times G$ of the group G. A regular Borel measure $\mu$ on $\mathbb{R}^{N+M} \times G^{*}=\mathbf{G}^{*}$ is said to be polynomially bounded, if the function

$$
\mathbf{G}^{*} \ni(\xi, \eta, \gamma) \mapsto\left(1+|\xi|^{2}+|\eta|^{2}\right)^{-n}
$$

is $|\mu|$-integrable for some $n \in \mathbb{N}$. Let $\mathscr{M}$ denote the set of all such measures. $\mathbf{M}=\left(\mu_{k l}\right)_{k, l \in \mathbf{N}} \in M(\infty ; \mathscr{M})$ is said to be positive, if each of the matrices $\left(\mu_{k l}(\Delta)\right)_{k, l=1}^{n}, n \in \mathbb{N}, \Delta \subseteq \mathbf{G}^{*}$ measurable, is nonnegative. $\mathbf{M} \in M(\infty ; \mathscr{M})$ defines a $G$-continuous matrix-valued distribution $\mathscr{F}=\left(f_{k l}\right)_{k, l \in N}$ by

$$
f_{k l, g}(\varphi)=\int_{G^{*}}[\gamma, g] \hat{\varphi}(\xi, \eta) d \mu_{k l}(g), \quad \varphi \in \mathscr{D}\left(\mathbb{R}^{N+M}\right),
$$

where

$$
\hat{\varphi}(\xi, \eta)=\int_{\mathbf{R}^{N+M}} \exp (-i \xi . x-i \eta . y) \varphi(x, y) d x d y .
$$


We call $\mathscr{F}$ the Fourier transform of $\mathrm{M}$ and write $\mathscr{F}=\widehat{\mathrm{M}}$. If $\mathscr{F}$ is a matrixvalued distribution on $2 \mathrm{C}$ and the above equality holds for $\varphi \in \mathscr{D}_{2 a}$, we write $\mathscr{F} \subseteq \widehat{\mathrm{M}}$. One easily checks that $\widehat{\mathbf{M}}$ is p.d. if $\mathbf{M}$ is positive. For $\mathbf{M} \in$ $M(\infty ; \mathscr{M})$ we can choose a fixed positive finite measure $\mu$ and measurable functions $a_{k l}$ on $\mathbf{G}^{*}$ such that $\mu_{k l}=a_{k l} \cdot \mu, k, l \in \mathbb{N}$. We will frequently use such a representation in the following. If $M$ is positive, we can choose the functions $a_{k l}$ such that all matrices $\left(a_{k l}(\xi, \eta, \gamma)\right)_{k, l=1}^{n},(\xi, \eta, \gamma) \in \mathbf{G}^{*}, n \in \mathbb{N}$, are nonnegative. We are now ready to state the following result.

Theorem 1. Let $G$ denote a separable locally compact abelian group and $\mathbf{G}=$ $\mathbf{R} \times \mathbb{R}^{M} \times G, M \in \mathbb{N} \cup\{0\}$ (i.e., $N=1$ ). Suppose that $\mathscr{F}=\left(f_{k l}\right)_{k, l \in \mathbb{N}}$ is a G-continuous p.d. matrix-valued distribution on $2 \mathbf{C}=(-2 a, 2 a) \times \mathbb{R}^{M} \times G$, $0<a \leq \infty$. Then there is a positive $\mathrm{M} \in M(\infty ; \mathscr{M})$ such that $\mathscr{F} \subseteq \widehat{\mathrm{M}}$.

Proof. 1. Let $G_{0}$ denote a countable dense subgroup of $G$. Consider the functional $T=T_{\mathscr{F}}$ associated with $\mathscr{F}$ (cf. Lemma 9). Since $T$ is positive, we can construct a Hilbert space $H$ as in $\S 6$. We define a unitary representation $U_{0}$ of $\mathbb{R}^{M} \times G_{0}$ in $H$ by

$$
U_{0}(y, g) J \Phi=J V(y, g) \Phi, \quad y \in \mathbb{R}^{M}, g \in G_{0}, \Phi \in E_{a} .
$$

Of course, $U_{0}$ is densely defined and straightforward calculation shows that the representation operators are unitary. Since $\mathscr{F}$ is $G$-continuous, the mapping $(y, g) \mapsto\left\langle U_{0}(y, g) J \Phi, J \psi\right\rangle$ is continuous for all $\Phi, \Psi \in E_{a}$. Since $U_{0}(y, g)$ is contained in the unit ball $B_{1}$ of $B(H)$ and since $J E_{a}$ is dense in $H, U_{0}$ is weakly continuous. Thus $U_{0}$ is uniformly weakly continuous on $\mathbb{R}^{M} \times G_{0}$. Since $B_{1}$ is weakly complete, there is a unique weakly continuous extension of $U_{0}$ to $\mathrm{R}^{M} \times G$. Obviously it has to be unitary, and we denote it by $U$. Hence $U$ becomes a strongly continuous unitary representation of $\mathbb{R}^{M} \times G$ in $H$.

2. We define a linear operator $A$ in $H$ by $D(A)=J E_{a}$ and

$$
A J J_{k, g} \varphi=J J_{k, g}\left(-i \frac{\partial}{\partial x} \varphi\right), \quad k \in \mathbb{N}, g \in G_{0}, \varphi \in \mathscr{D}_{a} .
$$

We show that $A$ is symmetric. First consider $\varphi, \psi \in \mathscr{D}_{a}$. One has

$$
(\partial \varphi / \partial x) * \psi=-\varphi *(\partial \psi / \partial x) .
$$

Thus

$$
\begin{aligned}
\left\langle A, J J_{k, g} \varphi, J J_{1, h} \psi\right\rangle & =T\left(J_{k, g}\left(-i \frac{\partial}{\partial x} \varphi\right) *\left(J_{1, h} \psi\right)^{*}\right) \\
& =T\left(\mathscr{J}_{k l, g-h}\left(\left(-i \frac{\partial}{\partial x} \varphi\right) * \psi^{*}\right)\right) \\
& =T\left(\mathscr{J}_{k l, g-h}\left(\varphi *\left(-i \frac{\partial}{\partial x} \psi\right)\right)\right) \\
& =\left\langle J J_{k, g} \varphi, A J J_{1, h} \psi\right\rangle
\end{aligned}
$$


for $k, l \in \mathbb{N}, g, h \in G_{0}$, and $\varphi, \psi \in \mathscr{D}_{a}$. By linearity we obtain the desired symmetry.

3. It is easy to see that the representation operators $U(y, g), y \in \mathbf{R}^{M}$, $g \in G_{0}$, commute with $A$ in the strong sense. By Proposition 11 we find a larger Hilbert space $\mathscr{H} \supseteq H$, a selfadjoint extension $\mathscr{A}$ of $A$ in $\mathscr{H}$, and an extension $\mathscr{U}$ of the representation $U$ which commutes strongly with $\mathscr{A}$. Now we use the direct integral decomposition of $\mathscr{H}$ according to Proposition 12.

$$
\mathscr{H}=\int_{\mathbf{R}^{M+1} \times G^{*}}^{\oplus} \mathscr{H}(\xi, \eta, \gamma) d \mu(\xi, \eta, \gamma) .
$$

According to Proposition 10 there are continuous linear mappings $J_{(\xi, \eta, \gamma)}$ : $E_{a} \mapsto \mathscr{H}(\xi, \eta, \gamma)$ such that

$$
J \Phi=\int_{\mathbf{R}^{M+1} \times G^{*}}^{\oplus} J_{(\xi, \eta, \gamma)} \Phi d \mu(\xi, \eta, \gamma) \text { for } \Phi \in E_{a} .
$$

4. Suppose that $\left\{\Phi^{n} ; n \in \mathbb{N}\right\}$ is a countable dense subset of $E_{a}$ and $\left\{Y_{n} ; n \in\right.$ $\mathbf{N}\}$ a countable dense subset of $\mathbf{R}^{M}$. Since

$$
\begin{aligned}
\mathscr{U}\left(y_{n}, g\right) J \Phi^{k} & =\int_{\mathbf{R}^{M+1} \times G^{*}}^{\oplus} \exp \left(-i \eta \cdot y_{n}\right)[\gamma, g] J_{(\xi, \eta, \gamma)} \Phi^{k} d \mu(\xi, \eta, \gamma) \\
& =J V\left(y_{n}, g\right) \Phi^{k}
\end{aligned}
$$

there is a $\mu$-null set $\mathscr{N}_{k, n, g}$ such that

$$
\begin{aligned}
& J_{(\xi, \eta, \gamma)} V\left(y_{n}, g\right) \Phi^{k}=\exp \left(-i \eta \cdot y_{n}\right)[\gamma, g] J_{(\xi, \eta, \gamma)} \Phi^{k} \\
& \quad \text { for }(\xi, \eta, \gamma) \in\left(\mathbf{R}^{M+1} \times G^{*}\right) \backslash \mathcal{N}_{k, n, g} .
\end{aligned}
$$

Setting $\mathscr{N}=\bigcup\left\{\mathcal{N}_{k, n, g} ; k, n \in \mathbb{N}, g \in G_{0}\right\}$, we obtain that the latter relation is true for all $n, k \in \mathbb{N}, g \in G_{0}$, and all $(\xi, \eta, \gamma) \in\left(\mathbb{R}^{M+1} \times G^{*}\right) \backslash \mathscr{N}$. Without loss of generality we assume that $\mathscr{H}(\xi, \eta, \gamma)=\{0\}$ for $(\xi, \eta, \gamma) \in \mathscr{N}$. Thus the relation is valid for all $(\xi, \eta, \gamma) \in \mathbf{R}^{M+1} \times G^{*}$. Since the mapping $y \mapsto V(y, g) \Phi$ is continuous for all $\Phi \in E_{a}$, we obtain

$$
\begin{aligned}
& J_{(\xi, \eta, \gamma)} V(y, g) \Phi=\exp (-i \eta \cdot y)[\gamma, g] J_{(\xi, \eta, \gamma)} \Phi \\
& \quad \text { for all }(\xi, \eta, \gamma) \in \mathbf{R}^{M+1} \times G^{*}, \Phi \in E_{a}, y \in \mathbb{R}^{M} \text {, and } g \in G_{0} .
\end{aligned}
$$

Changing the field of Hilbert spaces (if necessary) on a $\mu$-null set, we obtain similarly

$$
J_{(\xi, \eta, \gamma)}\left(-i \frac{\partial}{\partial x} \Phi\right)=\xi J_{(\xi, \eta, \gamma)} \Phi \text { for all }(\xi, \eta, \gamma) \in \mathbf{R}^{M+1} \times G^{*} \text { and } \Phi \in E_{a} \text {. }
$$

(Here $i(\partial / \partial x) \Phi$ is defined by $i(\partial / \partial x) J_{k, g} \varphi:=J_{k, g}(i(\partial / \partial x) \varphi), k \in \mathbb{N}, g \in$ $G_{0}$.) 
5. By [5, Proposition II.1.1], we have the following:

(i) The set $Z_{p}$ of $(\xi, \eta, \gamma) \in \mathbb{R}^{M+1} \times G^{*}$ such that the dimension $d(\xi, \eta, \gamma)$ of $\mathscr{H}(\xi, \eta, \gamma)$ is equal to $p$ is measurable.

(ii) There exists a sequence $\left(\mathbf{e}_{n}\right)_{n \in \mathbb{N}}$ of measurable vector fields possessing the following properties:

(a) if $d(\xi, \eta, \gamma)=\aleph_{0},\left(\mathbf{e}_{n}(\xi, \eta, \gamma)\right)_{n \in \mathbb{N}}$, is an orthonormal basis of $\mathscr{H}(\xi, \eta, \gamma)$,

(b) if $d=d(\xi, \eta, \gamma)<\aleph_{0},\left(\mathbf{e}_{n}(\xi, \eta, \gamma)_{n=1}^{d}\right.$ is an orthonormal basis of $\mathscr{H}(\xi, \eta, \gamma)$ and $\mathbf{e}_{n}(\xi, \eta, \gamma)=0$, if $n>d$.

6. Now the mapping $\mathscr{D}_{a} \ni \varphi \mapsto B(\varphi), B(\varphi):=\left\langle J_{(\xi, \eta, \gamma)} J_{l, 0} \varphi, \mathbf{e}_{n}(\xi, \eta, \gamma)\right\rangle$, defines a distribution $B \in \mathscr{D}_{a}^{\prime}$. Using (11) and (12) we compute

$$
\left(\frac{\partial}{\partial x} B\right)(\varphi)=-B\left(\frac{\partial}{\partial x} \varphi\right)=-i \xi B(\varphi) \text { for } \varphi \in \mathscr{D}_{a},
$$

i.e. $(\partial / \partial x) B=-i \xi B$ (in the distributive sense). Similarly we obtain $\left(\partial / \partial y_{j}\right) B$ $=-i \eta_{j} B, j=1, \ldots, M$. Thus $B$ is a regular distribution given by

$$
\begin{aligned}
B(\varphi) & =a_{l}^{n}(\xi, \eta, \gamma) \int_{\mathbf{R}^{M+1}} \exp (-i \xi x) \cdot \exp (-i \eta \cdot y) \varphi(x, y) d x d y \\
& =a_{l}^{n}(\xi, \eta, \gamma) \hat{\varphi}(\xi, \eta),
\end{aligned}
$$

where $a_{l}^{n}(\xi, \eta, \gamma)$ is a suitable constant. Hence we may comprise the result as follows:

$$
\left\langle J_{(\xi, \eta, \gamma)} J_{l, 0} \varphi, \mathbf{e}_{n}(\xi, \eta, \gamma)\right\rangle=a_{l}^{n}(\xi, \eta, \gamma) \hat{\varphi}(\xi, \eta)
$$

for $l, n \in \mathbb{N},(\xi, \eta, \gamma) \in \mathbb{R}^{M+1} \times G^{*}, \varphi \in \mathscr{D}_{a}$. Note that the function $(\xi, \eta, \gamma) \mapsto$ $a_{l}^{n}(\xi, \eta, \gamma)$ must be $\mu$-measurable since the left-hand side of (13) is $\mu$-measurable and since there are elements $\varphi$ of $\mathscr{D}_{a}$ such that the appropriate Fourier transform is different from zero on arbitrarily large compact subsets of $\mathbb{R}^{M+1}$.

7. Since

$$
J_{(\xi, \eta, \gamma)} V(0, g) J_{l, 0} \varphi=J_{(\xi, \eta, \gamma)} J_{l, g} \varphi=[\gamma, g] J_{(\xi, \eta, \gamma)} J_{l, 0} \varphi,
$$

we conclude from (13)

$$
\begin{aligned}
& \left\langle J_{(\xi, \eta, \gamma)} J_{l, g} \varphi, \mathbf{e}_{n}(\xi, \eta, \gamma)\right\rangle=a_{l}^{n}(\xi, \eta, \gamma)[\gamma, g] \hat{\varphi}(\xi, \eta) \\
& \quad \text { for } l, n \in \mathbb{N},(\xi, \eta, \gamma) \in \mathbb{R}^{M+1} \times G^{*}, g \in G_{0}, \varphi \in \mathscr{D}_{a} .
\end{aligned}
$$

Hence

$$
\begin{aligned}
\left\langle J_{(\xi, \eta, \gamma)}\right. & \left.J_{l, g} \varphi, J_{(\xi, \eta, \gamma)} J_{k, h} \psi\right\rangle \\
= & \sum_{n=1}^{\infty}\left\langle J_{(\xi, \eta, \gamma)} J_{l, g} \varphi, \mathbf{e}_{n}(\xi, \eta, \gamma)\right\rangle \cdot \overline{\left\langle J_{(\xi, \eta, \gamma)} J_{k, h} \psi, \mathbf{e}_{n} \overline{(\xi, \eta, \gamma)\rangle}\right.} \\
= & a_{l k}(\xi, \eta, \gamma)[\gamma, g-h] \hat{\varphi}(\xi, \eta) \overline{\hat{\psi}(\xi, \eta)} \\
= & a_{l k}(\xi, \eta, \gamma)[\gamma, g-h]\left(\hat{\varphi * \psi} \psi^{*}\right)(\xi, \eta),
\end{aligned}
$$


where the function $a_{l k}$ is defined by

$$
a_{l k}(\xi, \eta, \gamma)=\sum_{n=1}^{\infty} a_{l}^{n}(\xi, \eta, \gamma) \overline{a_{k}^{n}(\xi, \eta, \gamma)}
$$

Since

$$
\left\|J_{(\xi, \eta, \gamma)} J_{k, 0} \varphi\right\|^{2}=\sum_{n=1}^{\infty}\left|a_{k}^{n}(\xi, \eta, \gamma)\right|^{2}\left(\widehat{\varphi * \varphi^{*}}\right)(\xi, \eta)<\infty,
$$

we obtain that the sum in (15) is absolutely converging and $a_{l k}$ is a finite measurable function. Now we calculate for $g \in G_{0}$,

$$
\begin{aligned}
f_{k l, g}\left(\varphi * \psi^{*}\right) & =T\left(\left(J_{k, g} \varphi\right) *\left(J_{l, 0} \psi\right)^{*}\right)=\left\langle J J_{k, g} \varphi, J J_{l, 0} \psi\right\rangle \\
& =\int_{\mathbf{R}^{M+1} \times G^{*}}\left\langle J_{(\xi, \eta, \gamma)} J_{k, g} \varphi, J_{(\xi, \eta, \gamma)} J_{l, 0} \psi\right\rangle d \mu(\xi, \eta, \gamma) \\
& =\int_{\mathbf{R}^{M+1} \times G^{*}}[\gamma, g] a_{k l}(\xi, \eta, \gamma)\left(\hat{\varphi * \psi^{*}}\right)(\xi, \eta) d \mu(\xi, \eta, \gamma) .
\end{aligned}
$$

i.e. we have

$$
f_{k l, g}\left(\varphi * \psi^{*}\right)=\int_{\mathbf{R}^{M+1} \times G^{*}}[\gamma, g] a_{k l}(\xi, \eta, \gamma) \cdot\left(\widehat{\varphi * \psi^{*}}\right)(\xi, \eta) d \mu(\xi, \eta, \gamma)
$$

for all $g \in G_{0}, k, l \in \mathbb{N}, \varphi, \psi \in \mathscr{D}_{a}$.

8. Since for fixed $k \in \mathbb{N}$, the distribution $f_{k k, 0} \in \mathscr{D}_{2 a}^{\prime}$ is p.d. the representing measure $a_{k k} \cdot \mu$ must be polynomially bounded (cf. [17, p. $382 \mathrm{ff}$ ]. There the assertion was proved for radial distributions. The proof in the general case is quite similar.) It follows immediately from (15) that thus all measures $a_{k l} \cdot \mu:=$ $\mu_{k l}, k, l \in \mathbb{N}$, are polynomially bounded, i.e., $\mathbf{M}:=\left(\mu_{k l}\right)_{k, l \in N} \in M(\infty ; \mathscr{M})$, and that $M$ is positive. Moreover, we obtain that both sides in (16) define elements of $\mathscr{D}_{2 a}^{\prime}$, which coincide on the dense subset 1.h. $\left\{\varphi * \psi ; \varphi, \psi \in \mathscr{D}_{a}\right\}$ of $\mathscr{D}_{2 a}$ and are therefore equal. Consider an arbitrary test function $\varphi \in \mathscr{D}_{2 a}$ in place of $\left(\varphi * \psi^{*}\right)$ in (16). Then the left-hand side is continuous on $G_{0}$ (w.r.t. the topology induced from $G$ ) by assumption, whereas the continuity of the right hand side is easily checked. Thus this equality extends to all of $G$, which completes the proof.

Corollary 1. Let $G$ denote a locally compact abelian separable group. Suppose that $F:(-2 a, 2 a) \times G \rightarrow B(H), 0<a \leq \infty$, is a c.p.d. (operator-valued) function. Let $\left(\mathbf{e}_{n}\right)_{n \in N}$ denote an orthonormal basis in the separable Hilbert space $H$. Then there are

(i) a finite regular Borel measure $\mu$ on $\mathbf{R} \times G^{*}$ and

(ii) complex-valued functions $a_{k l} \in L^{1}\left(\mathbf{R} \times G^{*}, \mu\right), k, l \in \mathbb{N}$, with the property that the matrix $\left(a_{k l}(\xi, \gamma)\right)_{k, l \in N}$ is nonnegative for all $\xi \in \mathbb{R}, \gamma \in G^{*}$ such that

$$
\left\langle F(x, g) \mathbf{e}_{k}, \mathbf{e}_{l}\right\rangle \int_{\mathbf{R} \times G^{*}} \exp (-i \xi x)[\gamma, g] a_{k l}(\xi, \gamma) d \mu(\xi, \gamma)
$$

for all $x \in(-2 a, 2 a), g \in G, k, l \in \mathbb{N}$. 
Proof. Consider the $G$-continuous matrix-valued distribution $\mathscr{F}=\mathscr{F}$ (cf. $\S 4)$. Since $\mathscr{F}$ is $G$-continuous and positive definite, we obtain an integral representation according to Theorem 1 . Since the appropriate distributions are regular and since $\left\langle F(0,0) \mathbf{e}_{k}, \mathbf{e}_{k}\right\rangle=\int_{\mathbf{R} \times G^{*}} a_{k k}(\xi, \gamma) d \mu(\xi, \gamma)$ is finite, $a_{k k} \in$ $L^{1}\left(\mathbb{R} \times G^{*}, \mu\right)$ for $k \in \mathbb{N}$. It follows from (15) that then also $a_{k l} \in L^{1}\left(\mathbb{R} \times G^{*}, \mu\right)$ and that the matrix $\left(a_{k l}(\xi, \gamma)\right)_{k, l \in \mathbb{N}}$ is nonnegative for all $\xi \in \mathbb{R}$ and $\gamma \in G^{*}$. The representation (17) is now clear.

Corollary 2. Suppose that the assumptions of Corollary 1 are satisfied and that, in addition, $F(0,0)$ is a trace class operator. Then there are

(i) a finite regular Borel measure $\mu$ on $\mathrm{R} \times G^{*}$ and

(ii) a weakly measurable function $A: \mathbb{R} \times G^{*} \rightarrow B(H)$ with the property that $A(\xi, \gamma)$ is a nonnegative trace class operator and $\operatorname{tr}(A(\xi, \gamma))=1$ for $\mu$-a.a. $(\xi, \gamma) \in \mathbb{R} \times G^{*}$, such that

$$
\langle F(x, g) X, Y\rangle=\int_{\mathbf{R} \times G^{*}} \exp (-i \xi \cdot x)[\gamma, g]\langle A(\xi, \gamma) X, Y\rangle d \mu(\xi, \gamma)
$$

for all $x \in(-2 a, 2 a), g \in G, X, Y \in H$.

Proof. Since

$$
\begin{aligned}
\operatorname{tr}(F(0,0)) & =\sum_{n=1}^{\infty}\left\langle F(0,0) \mathbf{e}_{n}, \mathbf{e}_{n}\right\rangle \\
& =\sum_{n=1}^{\infty} \int_{\mathbf{R} \times G^{*}} a_{n n}(\xi, \gamma) d \mu(\xi, \gamma) \\
& =\int_{\mathbf{R} \times G^{*}} \sum_{n=1}^{\infty} a_{n n}(\xi, \gamma) d \mu(\xi, \gamma)<\infty,
\end{aligned}
$$

$a(\xi, \eta):=\sum_{n=1}^{\infty} a_{n n}(\xi, \gamma)$ is finite for all $(\xi, \gamma) \in\left(\mathbb{R} \times G^{*}\right) \backslash \mathscr{N}$, where $\mathscr{N}$ is a $\mu$-null set. Without loss of generality we assume that $a_{k l}(\xi, \gamma)=0$ for all $(\xi, \gamma) \in \mathscr{N}$ and $k, l \in \mathbb{N}$. All measures $a_{k l}(\xi, \gamma) d \mu(\xi, \gamma)$ are absolutely continuous w.r.t. the measure $a(\xi, \gamma) d \mu(\xi, \gamma)$, i.e. we may replace $\mu$ by the latter, i.e. we may assume in the following that $a(\xi, \gamma)=1 \quad \mu$-a.e. We define a linear operator on $D:=1 . h .\left\{\mathbf{e}_{n} ; n \in \mathbb{N}\right\}$ by $\left\langle A(\xi, \eta) \mathbf{e}_{k}, \mathbf{e}_{l}\right\rangle=a_{k l}(\xi, \eta)$. One easily checks that $\|X\|^{2} \geq\langle A(\xi, \eta) X, X\rangle \geq 0$ for $X \in D$. This implies that $A(\xi, \gamma)$ has a unique continuous extension, i.e. defines a bounded operator in $H$, which we denote by the same symbol. By construction, $A(\xi, \gamma)$ is even a trace class operator and $\operatorname{tr}(A(\xi, \gamma))=1 \mu$-a.e. Since $A(\xi, \gamma)$ is nonnegative on the dense set $D$, it is a nonnegative operator. (18) is obviously satisfied for $X, Y \in D$. But because of $1 \geq \operatorname{tr}(A(\xi, \gamma)) \geq\|A(\xi, \gamma)\|$ and since $\mu$ is finite $\left(\mu\left(\mathbb{R} \times G^{*}\right)=\operatorname{tr}(F(0,0))\right),(18)$ is true for all $X, Y \in H$.

Corollary 3. Let $G$ denote a separable topological abelian group and $H$ a separable Hilbert space. Then a c.p.d. function $F:(-2 a, 2 a) \times G \rightarrow B(H)$ has $a$ c.p.d. extension to $\mathbb{R} \times G$. 
Proof. Consider a countable dense subgroup $G_{0}$ of $G$, which will be equipped with the discrete topology for the moment. From Corollary 1, (17), we obtain an integral representation for $F_{1}:=F \mid(-2 a, 2 a) \times G_{0}$. We use (17) to extend $F_{1}$ to $\mathrm{R} \times G_{0}$. Denote this extension by $F_{2}$. Note that we define the operators $F_{2}(x, g)$ only on vectors of $D=1 . h .\left\{\mathbf{e}_{n} ; n \in \mathbb{N}\right\}$. But because of Lemma 1 , (i), all these operators are really bounded. By Lemma 3 there is a unique c.p.d. extension $F_{3}$ of $F_{2}$ to $\mathrm{R} \times G$, since $F_{2}$ coincides on a neighborhood of $(0,0)$ with $F_{1}$ and thus is continuous. Since $F_{3}$ is continuous, it coincides with $F$ on $(-2 a, 2 a) \times G$, i.e. $F_{3}$ is the desired extension.

Corollary 4. Suppose that $G$ is a locally compact separable abelian group, $H$ a separable Hilbert space, and $\left(\mathbf{e}_{n}\right)_{n \in N}$ a fixed orthonormal basis in $H$. Let $F=\left(f_{k l}\right)_{k, l \in N}$ denote a $G$-continuous positive definite matrix-valued distribution on $(-2 a, 2 a) \times \mathbf{R}^{M} \times G$. Then there are an operator-valued c.p.d. function $F: \mathbf{G}=$ $\mathbf{R}^{M+1} \times G \rightarrow B(H), \operatorname{tr}(F(0))<\infty$, a sequence of nonnegative integers $\left(r_{n}\right)_{n \in N}$, and a sequence of positive real numbers $\left(C_{n}\right)_{n \in N}$, such that

$$
f_{k l, g}(\varphi)=C_{k} C_{l} \int_{\mathbf{R}^{M+1}}\left\langle F(x, y, g) \mathbf{e}_{k}, \mathbf{e}_{l}\right\rangle\left((1-\Delta)^{r_{k}+r_{l}} \varphi\right)(x, y) d x d y,
$$

$k, l \in \mathbf{N}, g \in G, \varphi \in \mathscr{D}_{2 a}$. (Here we abbreviated the differential term $\partial^{2} / \partial x^{2}+$ $\partial^{2} / \partial y_{1}^{2}+\cdots+\partial^{2} / \partial y_{M}^{2}$ by $\left.\Delta.\right)$

Proof. Let $\mu_{k l}=a_{k l} \cdot \mu$ denote the measures as in Theorem 1. Since they are polynomially bounded, we may choose $r_{n} \in \mathbf{N}, c_{n}>0$, such that

$$
\int_{G^{*}}\left(1+|(\xi, \eta)|^{2}\right)^{-r_{n}} d \mu_{n n}(\xi, \eta, \gamma) \leq 2^{-n} C_{n} \text {. }
$$

The matrix $\left(b_{k l}\right)_{k, l \in N}$, where the entries $b_{k l}$ are functions on $\mathbf{G}^{*}$, defined by

$$
b_{k l}(\xi, \eta, \gamma)=C_{k}^{-1} C_{l}^{-1}\left(1+|(\xi, \eta)|^{2}\right)^{-r_{l}-r_{k}} a_{k l}(\xi, \eta, \gamma)
$$

is nonnegative and trace class for $\mu$-a.a. arguments. As in the proof of Corollary 2 , the relation

$$
\left\langle F(x, y, g) \mathbf{e}_{k} \mathbf{e}_{l}\right\rangle=\int_{\mathbf{R}^{M+1} \times G} \exp (-i \xi x) \exp (-i \eta y)[\gamma, g] b_{k l}(\xi, \eta, \gamma) d \mu(\xi, \eta, \gamma)
$$

defines a c.p.d. operator-valued function on $\mathbf{R}^{M+1} \times G$. For $\varphi \in \mathscr{D}_{2 a}$ we obtain from Theorem 1:

$$
\begin{aligned}
f_{k l, g}(\varphi) & =\int_{\mathbf{R}^{M+1} \times G}[\gamma, g] C_{k} C_{l}\left(1+|(\xi, \eta)|^{2}\right)^{r_{k}+r_{l}} b_{k l}(\xi, \eta, \gamma) \hat{\varphi}(\xi, \eta) d \mu(\xi, \eta, \gamma) \\
& =C_{k} C_{l} \int_{\mathbf{R}^{M+1} \times G^{*}}[\gamma, g] b_{k l}(\xi, \eta, \gamma)\left(\widehat{\left.(1-\Delta)^{r_{k}+r_{l}} \varphi\right)}(\xi, \eta) d \mu(\xi, \eta, \gamma),\right.
\end{aligned}
$$

which yields our assertion.

We conclude this chapter with the remark that Theorem 1 yields, in particular, the existence of a p.d. extension $\widehat{M}$ of $F$ to a $G$-continuous p.d. matrixvalued distribution on $\mathbf{G}$. 
10. N-RADIAL POSITIVE DEFINITE DISTRIBUTIONS ON A CYLINDER

Now we consider a group $\mathbf{G}=\mathbb{R}^{N} \times \mathbb{R}^{M} \times G$, where $N \in \mathbb{N}$ and $M \in$ $\mathbb{N} \cup\{0\}$ are arbitrary. Recall that $\mathscr{M}$ denotes the set of all regular polynomially bounded measures on $\mathbf{G}^{*}$. A measure $\mu \in \mathscr{M}$ is said to be $N$-radial, if it is invariant w.r.t. rotations in $\mathbb{R}^{N}=\left(\mathbb{R}^{N}\right)^{*}$. A matrix $\mathrm{M} \in M(\infty ; \mathscr{M})$ is called $N$-radial, if each of is entries is $N$-radial. By $\Omega_{N}: \mathbb{R}_{+} \rightarrow \mathbb{R}$ we denote the Bessel function with $\Omega_{N}(|x|)=\int_{|\xi|=1} \exp (-i \xi \cdot x) d \sigma_{N}(\xi), x \in \mathbb{R}^{N}$, where $\sigma_{N}$ is the surface measure of the unit sphere $\left\{\xi \in \mathbb{R}^{N} ;|\xi|=1\right\}$ normalized such that $\int d \sigma_{N}(\xi)=1$. For $\varphi \in \mathscr{D}\left(\mathbb{R}^{N+M}\right)$, we abbreviate

$$
\tilde{\varphi}\left(\tau^{1 / 2} \cdot \eta\right):=\int_{\mathbf{R}^{N+M}} \Omega_{N}\left(\tau^{1 / 2}|x|\right) \exp (-i \eta \cdot y) \varphi(x, y) d x d y .
$$

A measure $\mu$ on $\mathbb{R}_{+} \times \mathbb{R}^{M} \times G^{*}$ is said to be polynomially bounded, if there is some $n \in \mathbb{N}$ such that the function $(\tau, \eta, \gamma) \mapsto\left(1+\tau^{2}+|\eta|^{2}\right)^{-n}$ is $|\mu|$-integrable.

Note that the following theorem does not contain Theorem 1 as a special case. In particular, in case of $N=1$ it says that a symmetric (w.r.t. $x \mapsto-x$ ) p.d. distribution possesses a symmetric p.d. extension.

Theorem 2. Let $G$ denote a separable locally compact abelian group. Suppose that $F=\left(f_{k l}\right)_{k, l \in N}$ is a $G$-continuous $N$-radial p.d. matrix-valued distribution on $2 \mathbf{C}=S_{N}(2 a) \times \mathbb{R}^{M} \times G, 0<a \leq \infty$. Then there is a positive $N$-radial $\mathrm{M} \in M(\infty ; \mathscr{M})$ such that $F \subseteq \widehat{\mathrm{M}}$.

Proof. The proof is in large parts very similar to that of Theorem 1. So we will not repeat all arguments but refer to appropriate steps of the proof of that theorem.

1. Fix a countable dense subgroup $G_{0}$ of $G$ and consider the $N$-radial $G$-continuous functional $T=T_{F} \in \mathscr{E}_{a}^{\prime}$. Similarly as in Step 2 (Theorem 1), we obtain that

$$
T\left(J_{k, g}\left(-i \frac{\partial}{\partial x_{j}} \varphi\right) *\left(J_{1, h} \psi\right)^{*}\right)=T\left(J_{k, g} \varphi *\left(J_{1, h}\left(-i \frac{\partial}{\partial x_{j}} \psi\right)^{*}\right)\right)
$$

for $k, l \in \mathbb{N}, g, h \in G_{0}, \varphi, \psi \in \mathscr{D}_{a}$, and $j=1, \ldots, N$. Since $T$ is positive,

$$
T\left(\left(-i \frac{\partial}{\partial x_{j}} \boldsymbol{\Phi}\right) *\left(-i \frac{\partial}{\partial x_{j}} \boldsymbol{\Phi}\right)^{*}\right)=T\left(\left(-\frac{\partial^{2}}{\partial x_{j}^{2}} \boldsymbol{\Phi}\right) * \boldsymbol{\Phi}\right) \geq 0,
$$

where $\left(\partial / \partial x_{j}\right) \Phi, \Phi \in E_{a}$, is defined by $\left(\partial / \partial x_{j}\right) J_{k, g} \varphi=J_{k, g}\left(\partial / \partial x_{j}\right) \varphi$, $\varphi \in \mathscr{D}_{a}, k \in \mathbb{N}, g \in G_{0}$. Denote $\Delta_{x}=\partial^{2} / \partial x_{1}^{2}+\cdots+\partial^{2} / \partial x_{N}^{2}$. Thus we fix the following:

$$
T\left(\left(-\Delta_{x} \Phi\right) * \Phi^{*}\right) \geq 0 \text { for all } \Phi \in E_{a} .
$$

2. Now we use the functional $T$ and the nuclear space $E_{a}^{\#}$ to construct a Hilbert space $H^{\#}$ as in $\S 6$. Similarly as in Step 1 (Theorem 1) we obtain a strongly continuous unitary representation $U$ of $\mathbb{R}^{M} \times G$ in $H^{\#}$. 
3. Since $-\Delta_{x} E_{a}^{\#} \subseteq E_{a}^{\#}$, we may define an operator $A$ in $H^{\#}$ by $D(A)=J E_{a}^{\#}$ and $A J \Phi=J\left(-\Delta_{x} \Phi\right)$. (As before $J$ is the canonical imbedding of $E_{a}^{\#}$ in $H^{\#}$. By (19), $A$ is a symmetric nonnegative operator.

4. One easily checks that the unitary operators $U(y, g), y \in \mathbf{R}^{M}, g \in G_{0}$, commute strongly with $A$. By Proposition 11, the Friedrichs extension $\mathscr{A}$ commutes with all $U(y, g), y \in \mathbf{R}^{M}, g \in G$, in the strong sense. Now we use the direct integral representation of $H^{\#}$ according to Proposition 12:

$$
H^{\#}=\int_{\mathbf{R}_{+} \times \mathbf{R}^{M} \times G^{*}}^{\oplus} H(\tau, \eta, \gamma) d \mu(\tau, \eta, \gamma) .
$$

According to Proposition 10 there are continuous linear mappings $J_{(\tau, \eta, \gamma)}$ : $E_{a}^{\#} \rightarrow H(\tau, \eta, \gamma)$ such that

$$
J \Phi=\int_{\mathbf{R}_{+} \times \mathbf{R}^{M} \times G^{*}}^{\oplus} J_{(\tau, \eta, \gamma)} \Phi d \mu(\tau, \eta, \gamma) \text { for } \Phi \in E_{a}^{\#} .
$$

5. Arguing as in Step 4 (Theorem 1), we may assume without loss of generality that

$$
\begin{aligned}
& J_{(\tau, \eta, \gamma)} V(y, g) \Phi=\exp (-i \eta \cdot y)[\gamma, g] J_{(\tau, \eta, \gamma)} \Phi \\
& \quad \text { for all }(\tau, \eta, \gamma) \in \mathbf{R}_{+} \times \mathbf{R}^{M} \times G^{*}, \Phi \in E_{a}^{\#}, y \in \mathbf{R}^{M}, \text { and } g \in G_{0},
\end{aligned}
$$

and

(23) $J_{(\tau, \eta, \gamma)}\left(-\Delta_{x} \Phi\right)=\tau J_{(\tau, \eta, \gamma)} \Phi$ for all $(\tau, \eta, \gamma) \in \mathbb{R}_{+} \times \mathbf{R}^{M} \times G^{*}$ and $\Phi \in E_{a}^{\#}$.

6. We choose a sequence $\left(e_{n}\right)_{n \in N}$ of measurable vector fields as in Step 5 (Theorem 1) and consider the continuous linear functional $B$ on $\mathscr{D}_{a}^{\#}$ given by $B(\varphi):=\left\langle J_{(\tau, \eta, \gamma)} J_{1,0} \varphi, \mathbf{e}_{n}(\tau, \eta, \gamma)\right\rangle$. By Lemma 8 there is an unique $N$-radial distribution $B_{1} \in \mathscr{D}_{a}^{\prime}$ whose restriction to $\mathscr{D}_{a}^{\#}$ is $B$. Now $\varphi \mapsto B\left(-\Delta_{x} \varphi\right)$ defines a continuous linear functional on $\mathscr{D}_{a}^{\#}$. Since $-\Delta_{x} B_{1}$ is a radial distribution which extends the latter and since there is only one such extension, we obtain as in Step 6 (Theorem 1)

$$
-\Delta_{x} B_{1}=\tau B_{1} .
$$

In a similar way we obtain

$$
\frac{\partial}{\partial y_{j}} B_{1}=-i \eta_{j} B_{1}, \quad j=1, \ldots, M .
$$

The only $N$-radial distribution $B_{1}$ satisfying (24) and (25) is given by

$$
B_{1}(\varphi)=a_{1}^{n}(\xi, \eta, \gamma) \int_{\mathbf{R}^{N+M}} \Omega_{N}\left(\tau^{1 / 2}|x|\right) \exp (-i \eta \cdot y) .
$$

$\varphi(x, y) d x d y=a_{1}^{n}(\xi, \eta, \gamma) \tilde{\varphi}\left(\tau^{1 / 2}, \eta\right)$, where $a_{1}^{n}(\xi, \eta, \gamma)$ is a suitable constant. (cf. [17, p. 367]). Hence we have

$$
\begin{aligned}
\left\langle J_{(\tau, \eta, \gamma)} J_{l, 0} \varphi, \mathbf{e}_{n}(\tau, \eta, \gamma)\right\rangle & =a_{l}^{n}(\tau, \eta, \gamma) \tilde{\varphi}\left(\tau^{1 / 2}, \eta\right), \\
& \quad l, n \in \mathbb{N}, \varphi \in \mathscr{D}_{a}^{\#},(\tau, \eta, \gamma) \in \mathbf{R}_{+} \times \mathbb{R}^{M} \times G^{*} .
\end{aligned}
$$


As in Step 6 (Theorem 1) we can see that the functions $(\tau, \eta, \gamma) \mapsto a_{l}^{n}(\tau, \eta, \gamma)$ are measurable.

7. Now we obtain from (26) as in Step 7 (Theorem 1)

$$
\left\langle J_{(\tau, \eta, \gamma)} J_{l, g} \varphi, \mathbf{e}_{n}(\tau, \eta, \gamma)\right\rangle=a_{l}^{n}(\tau, \eta, \gamma)[\gamma, g] \tilde{\varphi}\left(\tau^{1 / 2}, \eta\right),
$$

$l, n \in \mathbb{N},(\tau, \eta, \gamma) \in \mathbb{R}_{+} \times \mathbb{R}^{M} \times G^{*}, g \in G_{0}, \varphi \in \mathscr{D}_{a}^{\#}$. By

$$
a_{l k}(\tau, \eta, \gamma)=\sum_{n=1}^{\infty} a_{l}^{n}(\tau, \eta, \gamma) \overline{a_{k}^{n}(\tau, \eta, \gamma)}
$$

we obtain measurable functions. Note that the sum in (28) converges absolutely for all arguments. The matrix $\left(a_{k l}(\tau, \eta, \gamma)\right)_{k, l \in \mathrm{N}}$ is nonnegative by construction. For the subsequent calculation we will use the identity

$$
\tilde{\varphi}\left(\tau^{1 / 2}, \eta\right) \overline{\tilde{\psi}\left(\tau^{1 / 2}, \eta\right)}=\left(\widetilde{\varphi * \psi^{*}}\right)\left(\tau^{1 / 2}, \eta\right) \text { for } \varphi, \psi \in \mathscr{D}_{a}^{\#}
$$

(cf. [17, (23)]). Thus we obtain for $g \in G_{0}$ and $\varphi, \psi \in \mathscr{D}_{a}^{\#}$,

$$
\begin{aligned}
f_{k l, g}\left(\varphi * \psi^{*}\right) & =T\left(\left(J_{k, g} \varphi\right) *\left(J_{l, 0} \psi\right)^{*}\right)=\left\langle J J_{k, g} \varphi, J J_{l, 0} \psi\right\rangle \\
& =\int_{\mathbf{R}_{+} \times \mathbf{R}^{M} \times G^{*}}\left\langle J_{(\tau, \eta, \gamma)} J_{k, g} \varphi, J_{(\tau, \eta, \gamma)} J_{l, 0} \psi\right\rangle d \mu(\tau, \eta, \gamma) \\
& =\int_{\mathbf{R}_{+} \times \mathbf{R}^{M} \times G^{*}}[\gamma, g] a_{k l}(\tau, \eta, \gamma)\left(\widetilde{\varphi * \psi^{*}}\right)\left(\tau^{1 / 2}, \eta\right) d \mu(\tau, \eta, \gamma) .
\end{aligned}
$$

Thus we may comprise the latter to

$$
f_{k l, g}\left(\varphi * \psi^{*}\right)=\int_{\mathbf{R}_{+} \times \mathbf{R}^{M} \times G^{*}}[\gamma, g] a_{k l}(\tau, \eta, \gamma) \cdot\left(\widetilde{\varphi * \psi^{*}}\right)\left(\tau^{1 / 2}, \eta\right) d \mu(\tau, \eta, \gamma)
$$

for all $g \in G_{0}, k, l \in \mathbb{N}, \varphi, \psi \in \mathscr{D}_{a}^{\#}$.

8. Since for fixed $k \in \mathbb{N}$ the distribution $f_{k k, 0}$ is positive definite, the appropriate representing measure is polynomially bounded (cf. [17, p. 382]). Using (28) we obtain that this is true for all measures $\left|a_{k l}(\tau, \eta, \gamma)\right| d \mu(\tau, \eta, \gamma)$. Hence both sides in (30) define functionals belonging to $\mathscr{D}_{2 a}^{\prime}$, which coincide on the subspace 1.h. $\left\{\varphi * \psi ; \varphi, \psi \in \mathscr{D}_{a}^{\#}\right\}$. Since it is dense in $\mathscr{D}_{2 a}^{\#}$ by Lemma 7, relation (30) holds for all $\varphi \in \mathscr{D}_{2 a}^{\#}$ in place of $\left(\varphi * \psi^{*}\right)$. Obviously we obtain a $N$-radial extension of the right-hand side, if arbitrary function of $\mathscr{D}_{2 a}$ are taken instead of $\left(\varphi * \psi^{*}\right)$. But since there is only one radial extension, it has to be equal to $f_{k l, g}$. Thus we have the equality

$$
f_{k l, g}(\varphi)=\int_{\mathbf{R}_{+} \times \mathbf{R}^{M} \times G^{*}}[\gamma, g] a_{k l}(\tau, \eta, \gamma) \cdot \tilde{\varphi}\left(\tau^{1 / 2}, \eta\right) d \mu(\tau, \eta, \gamma)
$$

for $g \in G_{0}, k, l \in \mathbb{N}$ and $\varphi \in \mathscr{D}_{2 a}$. For fixed $\varphi \in \mathscr{D}_{2 a}$, the left-hand side in (31) is continuous by assumption and the continuity of the right-hand side w.r.t. the topology induced from $G$ on $G_{0}$ is easy. Thus the equality is satisfied for all $g \in G$. Now we can rewrite (31) using a $N$-radial $\mu_{k l} \in \mathscr{M}$ instead of $a_{k l} \cdot \mu$, which completes the proof.

The proofs for the next four corollaries of Theorem 2 are quite similar to the proofs of the appropriate corollaries of Theorem 1 and therefore omitted. 
Corollary 5. Let $G$ denote a locally compact abelian separable group. Suppose that $F: S_{N}(2 a) \times G \rightarrow B(H), N \in \mathbb{N}, 0<a \leq \infty$, is a $N$-radial c.p.d. (operatorvalued) function. Let $\left(\mathbf{e}_{n}\right)_{n \in \mathbb{N}}$ denote an orthonormal basis of the separable Hilbert space $H$. Then there are

(i) a finite regular Borel measure $\mu$ on $\mathbf{R}_{+} \times G^{*}$ and

(ii) complex-valued functions $a_{k l} \in L^{1}\left(\mathbf{R}_{+} \times G^{*}, \mu\right), k, l \in \mathbb{N}$, with the property that the matrix $\left(a_{k l}(\tau, \gamma)\right)_{k, l \in \mathbb{N}}$, is nonnegative for all $\tau \in \mathbf{R}_{+}, \gamma \in G^{*}$ such that

$$
\left\langle F(x, g) \mathbf{e}_{k}, \mathbf{e}_{l}\right\rangle=\int_{\mathbf{R}_{+} \times G^{*}} \Omega_{N}\left(\tau^{1 / 2}|x|\right)[\gamma, g] a_{k l}(\tau, \gamma) d \mu(\tau, \gamma)
$$

for all $g \in G^{*}, x \in S_{N}(2 a)$, and $k, l \in \mathbb{N}$.

Corollary 6. Suppose that the assumptions of Corollary 5 are satisfied and that $F(0,0)$ is a trace class operator. Then there are

(i) a finite regular Borel measure $\mu$ on $\mathbf{R}_{+} \times G^{*}$ and

(ii) a weakly measurable function $A: \mathbf{R}_{+} \times G^{*} \rightarrow B(H)$ with the property that $A(\tau, \gamma)$ is a nonnegative trace class operator and $\operatorname{tr}(A(\tau, \gamma))=1$ for $\mu$-a.a. $(\tau, \gamma) \in \mathbf{R}_{+} \times G^{*}$, such that

$$
\langle F(x, g) X, Y\rangle=\int_{\mathbf{R}_{+} \times G^{*}} \Omega_{N}\left(\tau^{1 / 2}|x|\right)[\gamma, g]\langle A(\tau, \gamma) X, Y\rangle d \mu(\tau, \gamma)
$$

for all $x \in S_{N}(2 a), g \in G$, and $X, Y \in H$.

Corollary 7. Let $G$ denote a separable topological abelian group and $H$ a separable Hilbert space. Then each c.p.d. $N$-radial function $F: S_{N}(2 a) \times G \rightarrow B(H)$ has a c.p.d. $N$-radial extension to $\mathbf{R}^{N} \times G$.

Corollary 8. Let $G$ denote a separable locally compact abelian group, $H$ a separable Hilbert space, and $\left(\mathbf{e}_{n}\right)_{n \in \mathbb{N}}$ a fixed orthonormal basis in $H$. Suppose that $\mathscr{F}=\left(f_{k l}\right)_{k, l \in N}$ is a G-continuous p.d. N-radial matrix-valued distribution on $S_{N}(2 a) \times \mathrm{R}^{M} \times G$. Then there are an operator-valued c.p.d. $N$-radial function $F: \mathbf{R}^{N+M} \times G \rightarrow B(H), \operatorname{tr}(F(0,0,0))<\infty$, a sequence of nonnegative integers $\left(r_{n}\right)_{n \in \mathrm{N}}$, and a sequence of positive real numbers $\left(C_{n}\right)_{n \in \mathrm{N}}$, such that

$$
f_{k l, g}(\varphi)=C_{k} C_{l} \int_{\mathbf{R}^{N+M}}\left\langle F(x, y, g) \mathbf{e}_{k}, \mathbf{e}_{l}\right\rangle \cdot\left((1-\Delta)^{r_{k}+r_{l}} \varphi\right)(x, y) d x d y,
$$

$k, l \in \mathrm{N}, g \in G, \varphi \in \mathscr{D}_{2 a}$. Hence $\mathscr{F}$ has a G-continuous $N$-radial p.d. matrix-valued extension to $\mathbf{R}^{N+M} \times G$. (In this case, $\Delta$ is the abbreviation for $\partial^{2} / \partial x_{1}^{2}+\cdots+\partial^{2} / \partial x_{N}^{2}+\partial^{2} / \partial y_{1}^{2}+\cdots+\partial^{2} / \partial y_{M}^{2}$.)

We conclude our considerations with a result which is related to a theorem of Minlos, Sazonov, and Gross. We use the terminology from [13]. Suppose that $H$ is a separable real Hilbert space. Let $\mathbf{F}$ denote the set of all finite dimensional orthonormal projections in $H$. A subset $E$ of $H$ is called a cylindrical set, if $E=\{x \in H ; P x \in F\}$ for some $P \in \mathrm{F}$ and some Borel 
set $F \subseteq P H$. On the ring of cylindrical sets the Gaussian measure $\mu$ on $H$ is defined by $\mu(E)=(2 \pi)^{-n / 2} \int_{F} \exp \left(-\|x\|^{2} / 2\right) d x$, where $n=\operatorname{dim} P H$ and $E, F$, and $P$ are as above, and $d x$ denotes the usual Lebesgue measure on $P H$. A continuous seminorm $q$ on $H$ is called measurable, if for each $\varepsilon>0$ there is a $P_{0} \in \mathrm{F}$ such that $\mu(q(P x)>\varepsilon)<\varepsilon$ for all $P \in \mathbb{F}, P P_{0}=0$. Let $\tau_{m}$ denote the weakest vector space topology on $H$ such that all measurable seminorms are continuous.

Corollary 9 (cf. [3, p. 452]). Suppose that $H=H_{1} \oplus H_{2}, H_{1}$ finite dimensional, and that $B_{1}$ is an open ball around zero in $H_{1}$. If $k$ is a positive definite function on $B_{1} \oplus H_{2}$ with the following properties:

(i) $k$ is $\tau_{m}$-continuous at zero;

(ii) $k\left(x_{1}+x_{2}\right)=k\left(x_{1}^{\prime}+x_{2}\right)$ for all $x_{1}, x_{1}^{\prime} \in B_{1},\left\|x_{1}\right\|=\left\|x_{1}^{\prime}\right\|, x_{2} \in H$; then $k$ admits a representation $k(x)=\int_{H} \exp (i\langle x, \lambda\rangle) d \rho(\lambda)$, where $\rho$ is a finite positive Borel measure on $H$.

Proof. $H$ endowed with the topology $\tau_{m}$ is a separable topological group. By Corollary 7 there is an extension $k^{*}$ of $k$ to all of $H$, which is continuous (w.r.t. $\tau_{m}$ ) and p.d. Thus it is the characteristic function of a positive finite Borel measure $\rho$ on $H$ by the Theorem of Minlos, Sazonov, and Gross [13, Theorem 6.7], which completes the proof.

\section{REFERENCES}

1. W. Ambrose, Spectral resolution of groups of unitary operators, Duke Math. J. 11 (1944), 589-595.

2. Ju. M. Berezanskij, Expansions in eigenfunctions of self-adjoint operators, Naukova Dumka, Kiev, 1965. (Russian)

3. Ju. M. Berezanskij and I. M. Gali, Positive definite functions of infinitely many variables on a stratum, Ukrain. Mat. Z. 24 (1972), 435-464. (Russian)

4. Ju. M. Berezanskij and M. L. Gorbačuk, On extensions of positive definite functions of two variables, Ukrain. Mat. Z. 17, no. 5 (1965), 96-102. (Russian)

5. J. Dixmier, Von Neumann algebras, North-Holland, Amsterdam, 1981.

6. R. E. Edwards, Functional analysis, theory and applications, Holt, Rinehart, and Winston, New York, 1965.

7. J. Friedrich and L. Klotz, On extensions of positive definite operator-valued functions, Rep. Math. Phys. (to appear).

8. I. M. Gel' fand, A. G. Kostjučenko, Expansions in eigenfunctions of differential and other operators, Dokl. Akad. Nauk SSSR 103 (1955), 349-352. (Russian)

9. M. L. Gorbačuk, On representations of positive definite operator functions, Ukrain. Mat. Z. 17, no. 2, (1965) 29-45. (Russian)

10. P. E. T. Jørgensen, Self-adjoint extension operators commuting with an algebra, Math. Z. 169 (1979), 41-62.

11. G. Köthe, Topological vector spaces. II, Springer-Verlag, New York, Heidelberg and Berlin, 1979.

12. M. G. Krein, Sur le problème du prolongement des fonctions hermitiennes positives et continues, C. R. (Doklady) (N.S.) Acad. Sci. URSS 26 (1940), 17-22.

13. H.-H. Kuo, Gaussian measures in Banach spaces, Springer-Verlag, New York, 1975. 
14. B. Ja. Levin and I. E. Ovčarenko, Extensions of hermitian-positive functions defined on a strip, Teor. Funktsii Funktsional. Anal i Prilozhen. 5 (1967) 68-83. (Russian)

15. M. S. Livšic, On self-adjoint and skew-adjoint extensions of symmetric operators, Doctoral Thesis, Moscow, 1945. (Russian)

16. K. Maurin, Allgemeine Eigenfunktionsentwicklungen, Spektraldarstellung abstrakter Kerne, Eine Verallgemeinerung der Distributionen auf Lie'schen Gruppen, Bull. Acad. Polon. Sci. Séri. Sci. Math. Astronom. Phys. 7 (1959), 471-479.

17. A. E. Nussbaum, Integral representations of functions and distributions positive definite relative to the orthogonal group, Trans. Amer. Math. Soc. 175 (1973), 355-387.

18. __ On functions positive definite relative to the orthogonal group and the representation of functions as Hankel-Stieltjes transforms, Trans. Amer. Math. Soc. 175 (1973), 389-408.

19. A. Pietsch, Nukleare lokalkonvexe Räume, Akademie-Verlag, Berlin, 1965.

20. W. Rudin, The extension problem for positive definite functions, Illinois J. Math. 7 (1963), 532-539.

21. __ An extension theorem for positive definite functions, Duke Math. J. 37 (1970), 49-53.

22. K. Schmüdgen, On commuting unbounded self-adjoint operators. IV, Math. Nachr. 125 (1986), 83-102.

23. E. Thoma, Zur Reduktionstheorie in separablen Hilbert-Räumen, Math. Z. 67 (1957), 1-9.

Sektion Mathematik der Karl-Marx-Universität, Karl-MarX-Platz, Leipzig DDR7010, German Democratic Republic 